\title{
Oral films: current status and future perspectives
}

\section{II - Intellectual property, technologies and market needs}

Ana Filipa Borges ${ }^{1,2}$, Cláudia Silva $^{1}$, Jorge F. J. Coelho ${ }^{3}$, Sérgio Simões ${ }^{1,2}$

[1] Bluepharma, Indústria Farmacêutica, S.A.

[2] Faculty of Pharmacy, University of Coimbra

[3] CIEPQPF - Faculty of Sciences and Technology, University of Coimbra

\section{Contents}

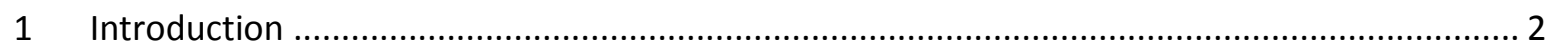

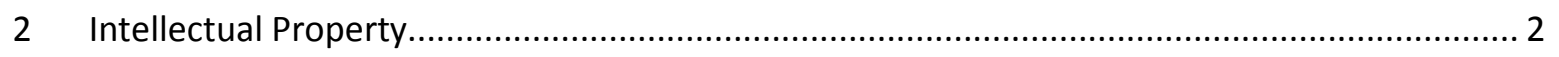

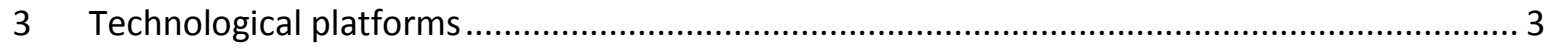

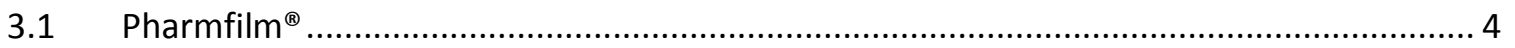

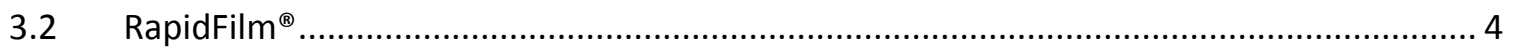

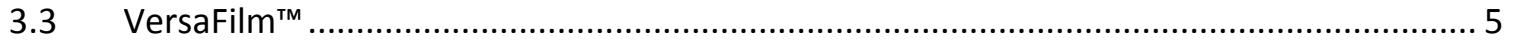

3.4 Orally and Adhesive Disintegrating Films .................................................................... 5

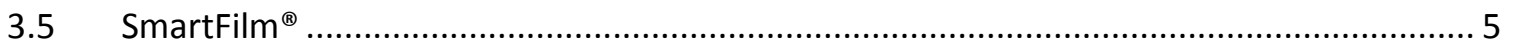

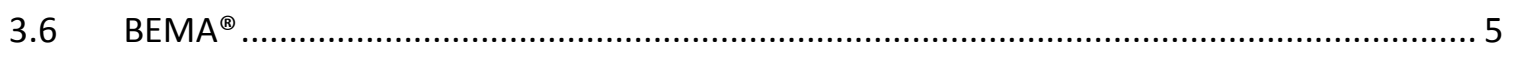

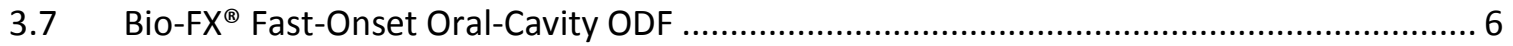

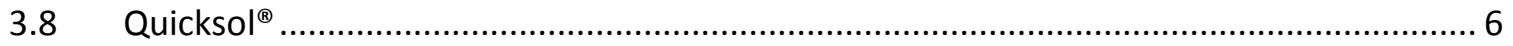

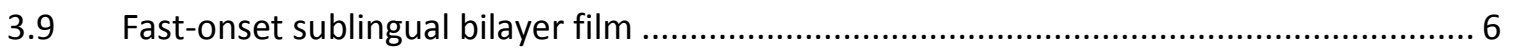

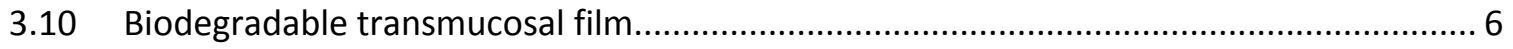

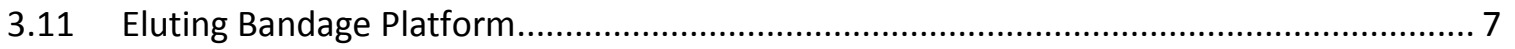

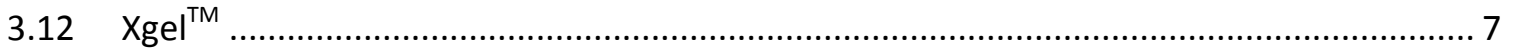

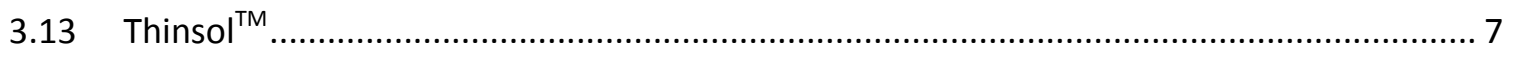

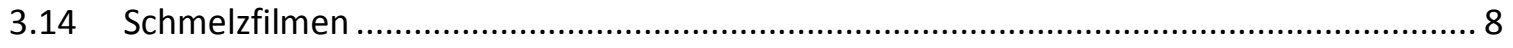

3.15 Others

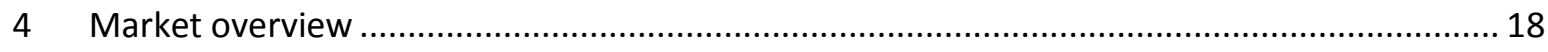

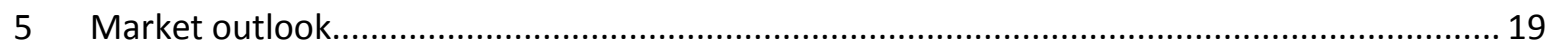




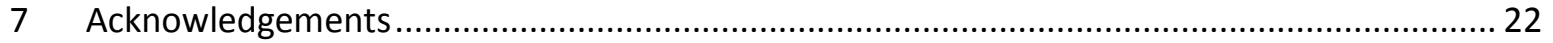

8 References. 23

\section{Introduction}

The oral route remains the most preferred for the general population [1]. It is easier, non-invasive, convenient and flexible, and generally oral formulations have a lower cost of production for the pharmaceutical companies. These facts justify why the oral delivery market holds $52 \%$ of the market share remaining the largest sector in the overall drug delivery market [2-4]. Although the majority of drugs are administered in the form of tablets and capsules, several groups of patients have serious swallowing difficulties. It is estimated that almost $28 \%$ of the general population have frequent problems in swallowing medicines that is often the cause of poor patient compliance [5]. This is commonly associated to dangerous tablets and capsules' modifications, such as splitting or crushing, related with dosage inaccuracy and drug therapy inefficiency or overdosing [6]. In order to overcome these issues, fast dissolving delivery systems are gaining considerable attention. Among them, oral films have emerged and have been dragged by this urgent market need.

There is no strong evidence or consensus about the date for the first reference of orodispersible delivery systems [7] but the most likely pioneer in the conception of orodispersible films was Deadman Frederic in 1960s [8]. Nevertheless, it remained just a concept until 2001 when Pfizer introduced in the market the major orodispersible film blockbuster, the Listerine ${ }^{\circledR}$ Pocket Packs ${ }^{\circledR}$ [9].

There is an evident trend that the pharmaceutical field is moving from the conventional and traditional to the innovative and patient-centered developments. There is also an increase demand of the authorities for knowledge, in order to improve the quality of the products, and the optimization and lean of the resources.

This section of the review highlights the Intellectual Property developed in this field, looking over the major players in the area, their platform technologies and all the commercial evolution through a summary market outlook and trends.

\section{Intellectual Property}

The drug delivery technology is an area with extensive intellectual property protection which is extremely important and required considering the high competitiveness of this fast-evolving field. There are a considerable number of institutions developing oral films, which can be easily confirmed by the constant growing number of patent applications. In fact, the increasing number of patents filled each year is impressive and more than 132 patent families have been identified and at least 30 companies / institutions are developing these technological platforms [10]. Until 2011, the majority of the patents were filled in the US and Japan, by the top players such as MonoSol and Kyukyu Pharmaceuticals Co.,LTD, with Europe gaining some ground in the recent years with LTS Lohman Therapy-Systems (LTS), Labtec Pharma, Hexal Pharmaceuticals and others. Additionally, LTS and MonoSol are clearly the major players with a broader technology coverage concerning the intellectual property, highlighting the diversified and fuelled research of these companies in the field [10]. At the moment according to the recent published Root Analysis report, 
MonoSol is the most prominent player in the oral thin films, with nine products already on the market based on its own technology [11].

Regarding intellectual property protection, an exhaustive search in free patent databases (google patents, Espacenet, WIPO) reveals that the composition patents are the larger slice in the overall patents filled. Among them, few are restricted to a specific therapy or drug substance, and the majority is therapeutically broader and focused in the composition of the technology, claiming essentially the film forming polymer(s), crucial for the matrix formation. The process patents have also some relevance, but only a few are restricted to a specific drug, therapy or method of use.

The most patented polymers are polysaccharides, including starch, cellulose and its derivatives (Figure 6). As already described in the part 1 of this review, these are two large groups of polymers that can be subdivided into subclasses according to the modifications and substituents added to the native natural polymer backbones.

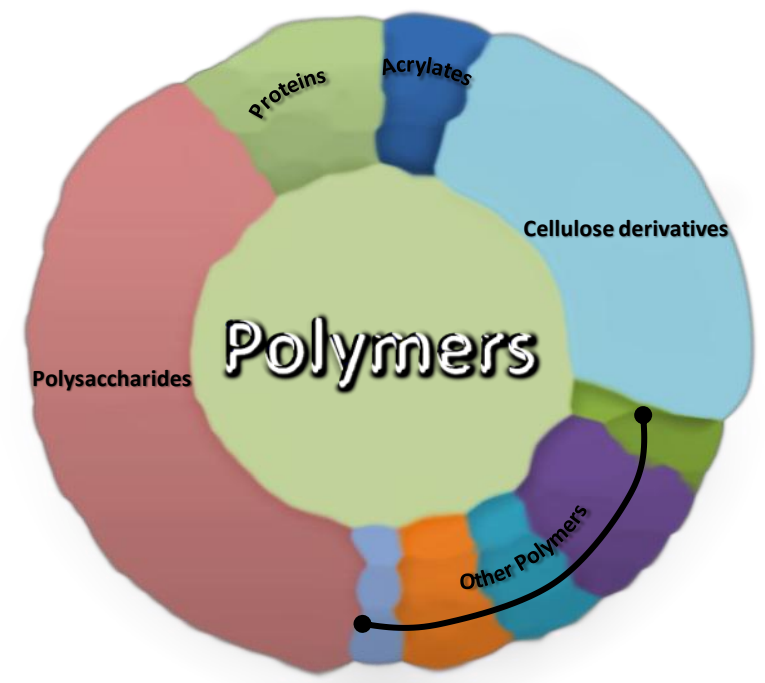

Figure 6 - Overall Scenario of polymers usage. The Polysaccharide group comprises starch derivatives, pectin, gums, dextrans and alginates; other polymers group includes polyvinyl and polyethylenoglycol polymers and co-polymers; the proteins groups consists of soy proteins, casein, zein, collagen and others; the acrylates groups refers mainly to methacrylate and polyacrylic polymers.

The use of the majority of hydrophilic polymers in formulations of oral films is already protected by several patents, restricting the possibility of developing formulations that do not infringe existing patents and capable of being protected by new ones. During the last years the development of new polymers suitable to this technological platform was scarce, leading to an increasing number of process patent applications, and patent formulations related with specific drug substances or therapies.

Furthermore, the difficulty in innovating in the formulation composition due to the small number of suitable excipients had probably contributed to new directions in this research field, such as the development of new manufacturing processes $[12,13]$ (see part 1 review), or the usage of oral films as drug delivery systems for biotechnology products (e.g. vaccines and insulin) [14-17].

\section{Technological platforms}

The majority of the top player companies referred above followed a similar pattern. Generally, a new and innovative technological platform is developed (like an oral film placebo) and then several drug candidates are evaluated and considered to be incorporated in the film. Obviously, 
this strategy implies necessarily the development of a versatile oral film platform, which in turn may suffer some modifications depending on the drug substance characteristics and the desired final dosage form performance.

Furthermore, it is common in this market segment the establishment of partnerships between oral film developers / manufacturers and other pharmaceutical companies researching new chemical entities, developing novel uses for existing drugs (repurposing) or companies looking for innovative formulations for their drugs (life-cycle management). This strategy is beneficial to share fixed expenses associated with the product licensing and marketing [18]. Therefore, two different major players may be distinguished in this field: the oral film platform developers, usually the technology owners, and the marketing partners.

Several oral film platforms have been already developed, the majority is listed on Table 4 and some are revised herein.

\subsection{Pharmfilm@}

MonoSol, one of the pioneer companies in the oral film industry owns a protected drug delivery technology, PharmFilm ${ }^{\circledR}$. MonoSol's film technology is supposed to be more stable and robust than other conventional dosage forms with a loading capacity up to $80 \mathrm{mg}$. The Pharmfilm ${ }^{\circledR}$ is a polymeric matrix based on polyethylene oxide and hydroxypropylmethyl cellulose, which normally is related with fast dissolution rates and rapid drug absorption [19]. However, MonoSol claims that this technological platform can be used for both fast dissolving system or buccal delivery. In fact, ondansetron hydrochloride had been successfully incorporated into the PharmFilm ${ }^{\circledR}$ technology as fast-dissolving system and others drug substances such as montelukast sodium, rizatriptan, escitalopram oxalate, donezepil hydrochloride and epinephrine are being considered or under development as oral quick release formulations [20-25]. Additionally, as previously referred, the Pharmfilm $^{\circledR}$ technology is also available as a slower release sublingual formulation (Suboxone ${ }^{\circledR}$ sublingual film) [25].

Moreover, MonoSol has established strategic partnerships to develop biotechnology sublingual and buccal films based on PharmaFilm ${ }^{\circledR}$ technology, such as anti-diabetic oral films or films to deliver a vaccine for universal flu. Together with Midatech, MonoSol has developed Nanoinsulin (insulin gold nanoparticles, MidaForm insulin) to incorporate in the MonoSol's PharmaFilm ${ }^{\circledR}$ buccal film technology, for the potential treatment of diabetes. In the beginning of 2013, this investigational medicinal product was listed as being in clinical development. Nevertheless, another buccal PharmaFilm ${ }^{\circledR}$ loaded with MidaForm nanoparticles, containing insulin and GLP-1, is also in preclinical development [14, 25, 26].

MonoSol in association with BiondVax Pharmaceuticals is developing a sublingual film formulation for vaccination, with the Multimeric-001 (M-001), for the potential prevention of universal influenza infection. It is expected that this type of formulation will allow the stability of the vaccine at room temperature $[15,27]$.

\subsection{RapidFilm @}

RapidFilm ${ }^{\circledR}$ is another patented technology developed by Labtec $\mathrm{GmBH}$. The Rapidfilm ${ }^{\circledR}$ is a fast dissolving thin film based on water soluble polymers, non-mucoadhesive, which can vary from single to multilayer design system. This oral film platform is based in a PVA-Starch mixture plasticized with a medium Mw PEG. The composition used allows its fast dissolution rate when in contact with the oral mucosa [28]. It is claimed that RapidFilm ${ }^{\circledast}$ can accommodate up to $30 \mathrm{mg}$ of the drug substances [20] [29]. The ondansetron Rapidfilm ${ }^{\circledR}$ was the first Rx oral film approval 
worldwide, but at the moment, there are at least three more Rapidfilm ${ }^{\circledR}$ products in the European market [30] (see table 4).

\subsection{VersaFilm $^{\mathrm{TM}}$}

VersaFilm ${ }^{\mathrm{TM}}$ technology was developed and patented by IntelGenx Technologies Corp. Initially developed as an edible film for the instant delivery of savoury flavours to food substrates, VersaFilm ${ }^{\mathrm{TM}}$ is now used as a system of choice for indications requiring an immediate onset of action. Thus, the company advances that VersaFilm ${ }^{\mathrm{TM}}$ 's disintegration time may be wrought from 30 seconds to 10 minutes, and it can be sublingual, depending on the intended application. The maximum drug load claimed is around 40mg. According to IntelGenx pipeline there are several drug substances in consideration or being incorporated in the Versafilm ${ }^{\mathrm{TM}}$ technology. However, only one has recently received a complete response letter from FDA, the rizatriptan VersaFilm ${ }^{\mathrm{TM}}$, an oral quick release film for migraine, developed together with RedHill Biopharma Ltd [31, 32].

\subsection{Orally and Adhesive Disintegrating Films}

KyuKyu Pharmaceuticals Co., Ldt is a Japanese company that also has its own oral film platform technology. Actually, KyuKyu have 2 different technologies the "Orally Disintegrating Film", which dissolves in 10 to 30 seconds and the "Adhesive and Disintegrating Film" that adheres to the oral mucosa and the disintegration time can vary between 30 minutes and 8 hours [33]. KyuKyu presents a large pipeline with several oral dispersible films in the market, mainly in the Asian market. Recently, it started to develop buccal films for the treatment of cancer-related pain and nicotine dependence. In collaboration with Nippon Kayaku, a buccal formulation of fentanyl is being developed and a phase II trial is being conducted [34]. Regarding to the nicotine mucoadhesive disintegrating film, it was in the fourth quarter of 2013 listed as being in lead optimization.

\subsection{SmartFilm ®}

Seoul Pharma has developed the SmartFilm ${ }^{\circledR}$ technology, an oral film with a high loading dose capacity, over $140 \mathrm{mg}$, capable of incorporating both hydrophilic and hydrophobic drugs, with unique taste masking technology and an eco-friendly manufacturing process (aqueous solution based). This South Korean pharmaceutical company launched Vultis ${ }^{\circledR}$ in the Korea market in 2012, a $140.45 \mathrm{mg}$ film formulation of sildenafil citrate. At the end of the same year, Seoul Pharma licensed it out to Pfizer which rebranded it as Viagra $[35,36]$. The Sidenafil SmartFilm ${ }^{\circledR}$ technology is a fast dissolving film composition that uses a combination of magnesium oxide and sodium hydroxide to mask the bitter taste of the drug substance. Seoul Pharma is currently seeking and researching other molecules to incorporate in its own oral film technology [37, 38].

\subsection{BEMA®}

BioDelivery Sciences International owns the worldwide rights of $\mathrm{BEMA}^{\circledR}$, bio-erodible mucoadhesive, drug delivery technology. This drug delivery technology consists in a bioerodible polymer film which adheres quickly to the oral mucosa (less than 5 seconds) with a backing layer that assures the unidirectional diffusion of the drug substance. This multilayer buccal film technology can rapidly deliver a dose of drug across the oral mucosa and is completely dissolved within 15 to 30 minutes. The $\mathrm{BEMA}^{\circledR}$ technology may be developed to incorporate several drug substances, especially if a quick onset of action is required, the oral administration dose is not optimal (low oral bioavailability) or if parental administration is not an option [39]. Onsolis ${ }^{\circledR}$, fentanyl buccal film, was the first product developed and marketed based on BEMA's technology, for the management of cancer pain in opioid-tolerant adults. It was launched in 2009 [40], but by March 
2012, the Onsolis ${ }^{\circledR}$ production had been temporarily closed in the US, due to FDA concerns regarding the manufacturing process [41]. In January 2014, it was announced that the re-launch of the product is planned to occur in the second half of 2014 [42, 43]. In Europe, the product was approved in October 2010 as Breakyl ${ }^{\circledR}$ [44]. Currently the BEMA ${ }^{\circledR}$ technology is being applied to improve the delivery of other therapies, as the opioid dependence with Bunavail ${ }^{\mathrm{TM}}$, previously referred. The base formulation of the BEMA ${ }^{\circledR}$ layers is very similar. Both the active and the backing layer are composed by hydroxypropyl cellulose, hydroxyethyl cellulose, but the active layer presents additional mucoadhesive polymers, as polycarbophil and carboxymethylcellulose sodium. Interestingly the sweetener and flavor are only present in the backing layer [19, 45].

\subsection{Bio-FX@ Fast-Onset Oral-Cavity ODF}

Another technology platform is the Bio-FX ${ }^{\circledR}$ Fast-Onset Oral-Cavity ODF from NAL Pharmaceuticals Ltd. Briefly, it is an oral film formulated with a Bio-FX ${ }^{\circledR}$ absorption enhancer system, which increases the absorption of the drug substances through the oral mucosa with the aim to improve the oral bioavailability of drugs by avoiding the first-pass metabolism and gastrointestinal degradation. This technology also incorporates a especial designed taste-masking system to improve taste and mouthfeel [46]. Currently, there are no available products on the market with this technology, but several are under development.

\subsection{Quicksol®}

Quicksol ${ }^{\circledR}$ technology is the oral film platform from SK Chemicals that can accommodate a wide variety of drug substances. According to the company's pipeline, several drug substances were loaded, but only two are already on the market, Montfree (Montelukast) ODF and Mvix-S (Mirodenafil) ODF [47]. Mvix-S is a thin, light and portable $50 \mathrm{mg}$ oral film, available since January 2012, with a mirodenafil rate absorption 16.7\% higher than Mvix tablet. Additionally, 15 days after its launch, Mvix-S sold over 1 billion units [48].

\subsection{Fast-onset sublingual bilayer film}

Cynapsus developed a fast-onset sublingual bilayer film of apomorphine, the APL-130277. The apomorphine in its neutral form (which may permit its fast mucosal absorption) is easily oxidized making difficult its incorporation in a film. Therefore, the apomorphine non-neutral form is loaded in one film layer, and a neutralizing agent is incorporated in other film layer, physically separated from each other. The neutralizing agent's layer dissolves quickly upon contact with saliva, allowing a fast reaction with the drug substance for a rapidly absorption. Clinical trials demonstrated that the maximum blood levels were reached within 20 minutes of administration, in the majority of subjects, and that it has a good local tolerability (no irritation). The submission of a FDA 505(b)(2) NDA is expected to 2016, since Cynapsus estimate to complete efficacy and safety studies by the end of 2014 and 2015, respectively. This sublingual formulation had already proved to work in the most severe cases of Parkinson's disease. Moreover, it may also present patient benefits and competitive advantages over the subcutaneous injection available and the inhaled and pulmonary approaches that are still in early development stages. According to their patent application the main polymer may be a cellulose, as HEC and / or a modified starch as maltodextrins, or even a mixture thereof [49].

\subsection{Biodegradable transmucosal film}

In the first quarter of 2005, Auxilium Pharmaceuticals had licensed an oral drug delivery system, based on the PharmaForm technology after their drug delivery platform acquisition. This platform is a biodegradable transmucosal film that adheres to the upper gum, preferentially above the back 
molar, and after that it completely dissolves. PharmaForm technology may allow a more effective delivery of the substances through a higher rate of drug absorption, contributing to achieve the same therapeutic levels with lower doses when compared with the conventional dosage form, shorter onset of action, reduction of first pass metabolism and probably less frequent dosing. Auxilium was using this technology platform to incorporate drug substances for the treatment of overactive bladder, management of pain and androgen replacement therapy. According to the company information, the overactive bladder transmucosal film candidate was supposed to be moving to phase II studies, after being demonstrated that oxybutynin could be administered using the transmucosal film, but no development has been recently reported. Similarly, Fentanyl Pharmaform film, which was in phase I development in 2011, has no recent updated information. Regarding to testosterone transmucosal film (TestoFilm), it was in a phase III trial in the beginning of 2006. However, in the last quarter of the same year Auxilium discontinued the development claiming that the formulation would not be commercially viable $[50,51]$.

\subsection{Eluting Bandage Platform}

Pharmedica has an innovative and patented oral film platform, the Eluting Bandage Platform. This is a multiple characteristic platform that can be used as single or multiple layer, with fast or slow disintegration time and for combined or protective treatment. Eluting Bandage Platform is a multipurpose and multi-functional device that can be used for a large range of products, from fresh breathers to prescription products. Pharmedica was developing oral formulations of insulin for the potential treatment of diabetes which had a launch predicted date for 2013 . However, no more information is available, but according to the company's website the insulin, together with cannabinoids, is still listed as a potential product for the Eluting Bandage platform [52].

\section{$3.12 \mathrm{Xgel}^{\mathrm{TM}}$}

$\mathrm{Xgel}^{\mathrm{TM}}$ film's technology is the basis of Meldex International intellectual property, used in all its film systems: Soluleaves ${ }^{\mathrm{TM}}$, Foamburst ${ }^{\mathrm{TM}}$ and Wafertab $^{\mathrm{TM}}$. Soluleaves ${ }^{\mathrm{TM}}$ platform can be designed for fast dissolving release or to adhere to the oral mucosa for a slow release of the drug substance. The Foamburst ${ }^{\mathrm{TM}}$ is a variant of the previous technology where an inert gas is passed during the film's manufacture resulting in a honeycomb structure that controls the dissolution rate of the drug substance contributing to a novel mouth sensation. In turn, the Wafertab ${ }^{\text {TM }}$ platform is prepared from a placebo $\mathrm{Xgel}^{\mathrm{TM}}$ film in which the drug substance is added afterwards, thus preventing its exposure to unnecessary heat and moisture. This technology allows the manufacture of unstable drugs and the preparation of multilayer films [20,53]. In 2007, Meldex was developing nicotine Soluleaves ${ }^{\mathrm{T} M}$, but no recent development has been reported. According to the patent information this is a cellulose derivative based film [54].

\subsection{ThinsolTM}

BioEnvelop (or Paladin Labs) has also its own patented technology, the Thinsol ${ }^{\mathrm{TM}}$, an oral film based on enzymatically digested carboxy methyl cellulose. This platform is a fast dissolving film (from five to 30 seconds) that allows a drug loading up to $60 \%$ and can be used to incorporate heat sensitive drugs, since it can be dried at low temperatures [20,55-57].

Interestingly, the NeuroHealing Pharmaceuticals Inc. developed an intra-oral slow dissolving mucoadhesive thin film based on the original formulation of Listerine Pocket Packs ${ }^{\circledR}$. This modified oral film was developed to incorporate $1 \mathrm{mg}$ of tropicamide for sialorrhea treatment. In fact, this buccal film platform, designated by the code name NH004, has two main modifications: additional mucoadhesive properties and a slower dissolution capability. Therefore the $\mathrm{NHOO4}$ easily adheres to the oral mucosa to dissolve slowly, over a period of 60-90 minutes, so the drug can be absorbed 
locally near the submandibular salivary gland [58]. Currently a phase II clinical trial is being conducted in order to evaluate the safety and efficacy of tropicamide thin films in hypersalivation Parkinson's patients treatment [59].

\subsection{Schmelzfilmen}

The "Schmelzfilmen", or melting film, was developed by Hexal and has currently four marketed products: olanzapine, sildenafil, donepezil and risperidone [60-62]. Although there are some composition variations between the four formulations, they are mainly cellulose based films. The olanzapine oral film commercially available presents Ethylcellulose as main film forming polymer, plasticized with dibutylsebacate, and apparently according to the patent claims the HPMC is essentially used as gelling agent, although the amounts described may indicate that it can also be used as a film-forming polymer [63-67].

\subsection{0thers}

Additionally, other attention-grabbing technology is Nutra3 Complex ${ }^{\circledast}$, a fast dissolving strip with a high loading capacity around $250 \mathrm{mg}$ per film. Unfortunately, there are no recent reports regarding this product [68].

Also, FFT Medical presented its own transmucosal drug delivery technology based on a alginate polymeric film, the FFT trans-mucosal film. The company claims that this technology allows delivering a wide variety of substances by a rapid and consistent absorption through the oral mucosa surface directly into the bloodstream. They also refer that the drug dissolution is performed in a controlled rate to avoid the release to the saliva [69].

Additional information about these technology platforms and others are summarized in table 4 . 
Table 4-Oral Films' technology platforms, their owners or developers, related patents and associated marketed products. * means that there is no specific information about the designation and /or status of the technology / product.

\begin{tabular}{|c|c|c|c|c|c|c|c|c|c|}
\hline \multirow{2}{*}{$\begin{array}{l}\text { Brand name / } \\
\text { Designation }\end{array}$} & \multirow{2}{*}{$\begin{array}{l}\text { Owner / } \\
\text { Originator } \\
\text { Company }\end{array}$} & \multirow[t]{2}{*}{ Patent (s) } & \multirow{2}{*}{$\begin{array}{l}\text { Active Companies / } \\
\text { Partner / Distributor }\end{array}$} & \multirow{2}{*}{$\begin{array}{l}\text { Commercial } \\
\text { products }\end{array}$} & & & \multirow{2}{*}{$\begin{array}{l}\text { Oral Film } \\
\text { Type }\end{array}$} & \multirow[t]{2}{*}{ Polymer } & \multirow[t]{2}{*}{ Ref. } \\
\hline & & & & & $\begin{array}{c}\text { Drug substance (if } \\
\text { any) }\end{array}$ & Phase / Status & & & \\
\hline \multirow[t]{4}{*}{ Buccal Wafer } & \multirow[t]{4}{*}{ LTS Lohman } & US-07407669 B2 & Pfizer & $\begin{array}{l}\text { Listerine }{ }^{\circledR} \text { Pocket } \\
\text { Packs }^{\circledR}\end{array}$ & & Launched & \multirow[t]{4}{*}{ dispersible } & & \multirow[t]{4}{*}[70,71]{} \\
\hline & & & McNeil-PPC & Sudafed PE ${ }^{T M}$ & Phenylephrine & Discontinued & & & \\
\hline & & & McNeil-PPC & Benadryl ${ }^{\circledR}$ & Diphemhydramine hcL & Discontinued & & & \\
\hline & & & GlaxoSmithKline & $\begin{array}{l}\text { NiQuitin Strips } 2.5 \mathrm{mg} \\
\text { Oral Film }\end{array}$ & Nicotine & Launched & & & \\
\hline \multirow[t]{9}{*}{ VersaFilm $^{\mathrm{TM}}$} & Intelgenx & & RedHill Biopharma & & Rizatriptan film & Approved by FDA & dispersible & & \multirow[t]{9}{*}[31,32,72]{} \\
\hline & $\begin{array}{l}\text { Technology } \\
\text { Corp. }\end{array}$ & $\begin{array}{l}\text { US- } \\
20110136815\end{array}$ & & & Tadalafil film & $\begin{array}{l}\text { Phase } 2 \text { Clinical Pilot } \\
\text { study planed for Q1 } \\
2014\end{array}$ & dispersible & & \\
\hline & & & & & INT0020 Insomnia & Phase 2 Clinical & dispersible & & \\
\hline & & & & & $\begin{array}{l}\text { INT-0022; anti- } \\
\text { psychotic agent }\end{array}$ & Phase 2 Clinical & dispersible & & \\
\hline & & & & & INT-0023 - Allergy & Phase 1 Clinical & dispersible & & \\
\hline & & & & & $\begin{array}{l}\text { INT-0025 - Prostate } \\
\text { hyperplasia }\end{array}$ & Phase 1 Clinical & dispersible & & \\
\hline & & & & & $\begin{array}{l}\text { INT0031 Benign } \\
\text { Prostatic } \\
\text { Hyperplasia }\end{array}$ & Pilot study & dispersible & & \\
\hline & & & & & $\begin{array}{l}\text { INT0030 - Animal } \\
\text { health Vetafilm }\end{array}$ & Pilot study & dispersible & & \\
\hline & & & & & INT0036 - CNS & Discovery & dispersible & & \\
\hline Thinsol $^{\mathrm{TM}}$ & $\begin{array}{l}\text { Paladin Labs } \\
\text { BioEnvelop's's }\end{array}$ & $\begin{array}{l}\text { WO- } \\
2009055923\end{array}$ & & & & & dispersible & & $\begin{array}{l}{[20,55,56][57,} \\
73]\end{array}$ \\
\hline
\end{tabular}




\begin{tabular}{|c|c|c|c|c|c|c|c|c|c|}
\hline \multirow{2}{*}{$\begin{array}{l}\text { Brand name / } \\
\text { Designation }\end{array}$} & \multirow{2}{*}{$\begin{array}{l}\text { Owner / } \\
\text { Originator } \\
\text { Company }\end{array}$} & \multirow[t]{2}{*}{ Patent (s) } & \multirow{2}{*}{$\begin{array}{l}\text { Active Companies / } \\
\text { Partner / Distributor }\end{array}$} & \multirow[t]{2}{*}{ Products } & & & \multirow{2}{*}{$\begin{array}{l}\text { Oral Film } \\
\text { Type }\end{array}$} & \multirow[t]{2}{*}{ Polymer } & \multirow[t]{2}{*}{ Ref } \\
\hline & & & & & $\begin{array}{l}\text { Drug substance (if } \\
\text { any) }\end{array}$ & Phase / Status & & & \\
\hline \multirow[t]{17}{*}{ Pharmfilm ${ }^{\circledR}$} & \multirow[t]{17}{*}{$\begin{array}{l}\text { MonoSol Rx } \\
\text { LLC }\end{array}$} & $\begin{array}{l}\text { U.S. patent No. } \\
7,824,588\end{array}$ & C.B. Fleet Company & $\begin{array}{l}\text { Pedia Lax }{ }^{\circledR} \text { Quick } \\
\text { Dissolve Strips }\end{array}$ & Sennosides & Discontinued & & \multirow{17}{*}{$\begin{array}{l}\text { Polyethylen } \\
\text { e oxide and } \\
\text { HPMC }\end{array}$} & \multirow{14}{*}{$\begin{array}{l}{[14,} \\
20-25] \\
{[70],[7} \\
4-82]\end{array}$} \\
\hline & & WO-2011017483 & $\begin{array}{l}\text { Reckitt Benckiser } \\
\text { Pharmaceuticals }\end{array}$ & $\begin{array}{l}\text { Suboxone }{ }^{\circledR} \text { Sublingual } \\
\text { Film }\end{array}$ & $\begin{array}{l}\text { Buprenorphine } \\
\text { Hydrochloride + } \\
\text { Naloxone } \\
\text { Hydrochloride }\end{array}$ & Launched & dispersible & & \\
\hline & & & \multirow[t]{2}{*}{ Prestige Brands } & Chloraseptic $^{\circledR}$ & Benzocaine & Discontinued & dispersible & & \\
\hline & & & & $\begin{array}{l}\text { Little cold sore throat } \\
\text { strip }\end{array}$ & $\begin{array}{l}\text { (Pectin) + Ascorbic } \\
\text { acid }\end{array}$ & Discontinued & dispersible & & \\
\hline & & WO-2013019187 & KemPharm's & & Methylphenidate & Discovery & dispersible & & \\
\hline & & WO-2008098151 & & & prodrug + ligand & & & & \\
\hline & & WO-2012040262 & MonoSol Rx LLC & & Montelukast Sodium & Clinical & dispersible & & \\
\hline & & & MonoSol Rx LLC & & $\begin{array}{l}\text { Diphenhydramine } \\
\text { hydrochloride }\end{array}$ & * & dispersible & & \\
\hline & & & MonoSol Rx LLC & & Escitalopram & $\begin{array}{l}\text { No Development } \\
\text { Reported }\end{array}$ & dispersible & & \\
\hline & & & MonoSol Rx LLC & & Rizatriptan & Discovery & dispersible & & \\
\hline & & & MonoSol Rx LLC & & Epinephrine & $\begin{array}{l}\text { No Development } \\
\text { Reported }\end{array}$ & dispersible & & \\
\hline & & WO-2013026002 & MonoSol Rx LLC & & Testosterone & Discovery & & & \\
\hline & & WO-2012177326 & $\begin{array}{l}\text { Midatech MidaSol } \\
\text { Therapeutics }\end{array}$ & & $\begin{array}{l}\text { Insulin nanoparticles } \\
\text { (MidaForm insulin) }\end{array}$ & Phase 1 Clinical & buccal & & \\
\hline & & & BiondVax & & Multimeric-001 & Discovery & dispersible & & \\
\hline & & $\begin{array}{l}\text { WO-2004066986 } \\
\text { WO-2006031209; } \\
\text { WO-03030881; WO- } \\
2012040262\end{array}$ & $\begin{array}{l}\text { MonoSol Rx LLC; Vestiq } \\
\text { Pharmaceuticals Inc }\end{array}$ & Zuplenz $^{\circledR}$ & $\begin{array}{l}\text { Ondansetron } \\
\text { Hydrochloride }\end{array}$ & Launched & dispersible & & \\
\hline & & & MonoSol/Midatech & & GLP-1 peptides & Discovery & buccal & & \\
\hline & & WO-2011124570 & $\begin{array}{l}\text { APR Applied Pharma; } \\
\text { MonoSol Rx LLC; Tesa } \\
\text { Labtec GmbH }\end{array}$ & & Zolmitriptan & Launched & dispersible & & [83] \\
\hline
\end{tabular}




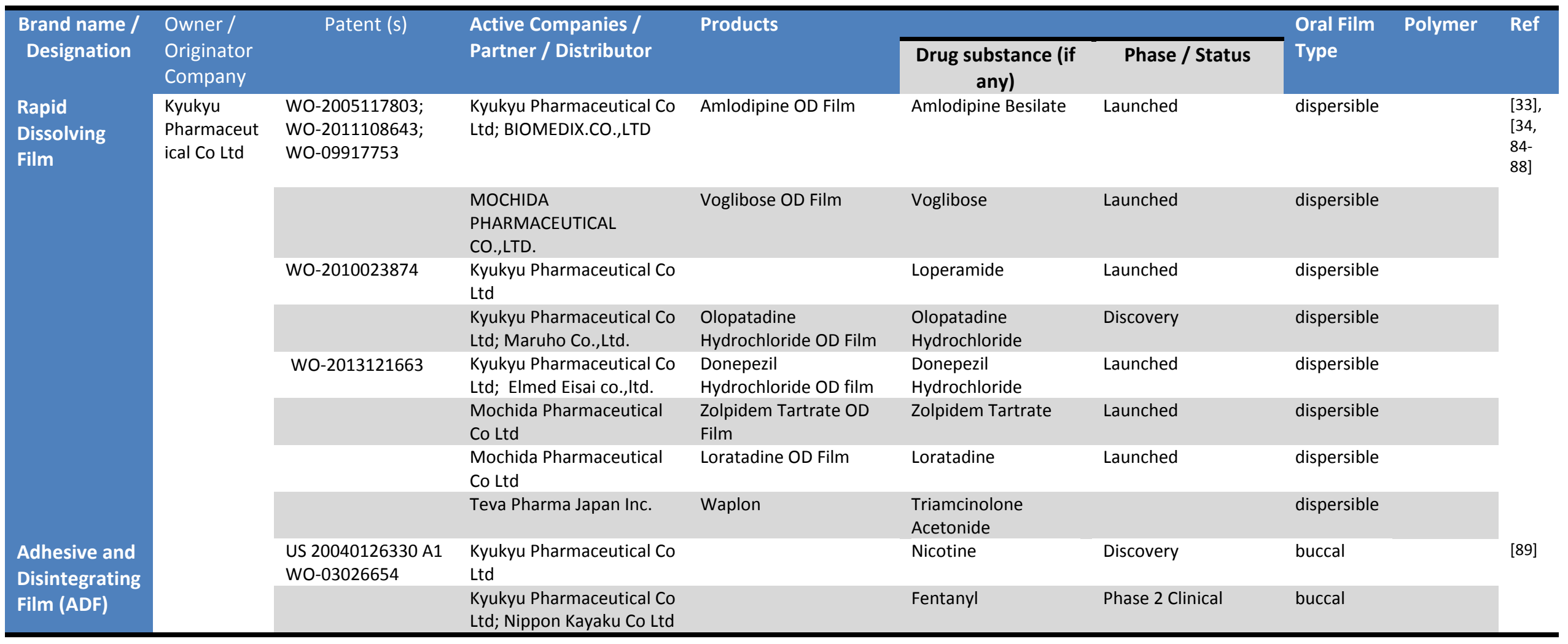




\begin{tabular}{|c|c|c|c|c|c|c|c|c|c|}
\hline \multirow{2}{*}{$\begin{array}{l}\text { Brand name / } \\
\text { Designation }\end{array}$} & \multirow{2}{*}{$\begin{array}{l}\text { Owner / } \\
\text { Originator } \\
\text { Company }\end{array}$} & \multirow[t]{2}{*}{ Patent (s) } & \multirow{2}{*}{$\begin{array}{l}\text { Active Companies / } \\
\text { Partner / Distributor }\end{array}$} & \multirow[t]{2}{*}{ Products } & & & \multirow{2}{*}{$\begin{array}{l}\text { Oral Film } \\
\text { Type }\end{array}$} & \multirow[t]{2}{*}{ Polymer } & \multirow[t]{2}{*}{ Ref } \\
\hline & & & & & $\begin{array}{l}\text { Drug substance (if } \\
\text { any) }\end{array}$ & Phase / Status & & & \\
\hline \multirow{11}{*}{$\begin{array}{l}\text { Dissolvable } \\
\text { Film } \\
\text { Technology } \\
\text { RapidFilm }{ }^{\circledR}\end{array}$} & \multirow[t]{3}{*}{ ARx } & & \multirow[t]{3}{*}{ Novartis Consumer Health } & Gas- $X^{\circledast}$ & Simethicone & Launched & dispersible & \multirow{11}{*}{$\begin{array}{l}\text { PVA } \\
\text { Starch } \\
\text { Medium } \\
\text { MwPEG }\end{array}$} & \multirow[t]{3}{*}{ [70] } \\
\hline & & & & Theraflu ${ }^{\circledR}$ Thin Strips ${ }^{\circledR}$ & Dextromethorphan & Discontinued & dispersible & & \\
\hline & & & & Triaminic $^{\circledR}$ Thin Strips ${ }^{\circledast}$ & Phenylephrine & Discontinued & dispersible & & \\
\hline & \multirow{8}{*}{$\begin{array}{l}\text { Labtec } \\
\text { GmBH / APR } \\
\text { Applied } \\
\text { Pharma } \\
\text { Research }\end{array}$} & $\begin{array}{l}\text { WO-2008040534; } \\
\text { WO-2009043588 }\end{array}$ & \multirow{4}{*}{$\begin{array}{l}\text { Norgine (Europe and } \\
\text { Middle East, Africa and } \\
\text { Australasia) / } \\
\text { SciClone Pharmaceuticals, } \\
\text { Inc (China and Vietnam) / } \\
\text { Takeda Canada (Canada) / } \\
\text { Monosol RX § }\end{array}$} & \multirow{4}{*}{$\begin{array}{l}\text { Setofilm }^{\circledast} / \\
\text { Ondansetron } \\
\text { Rapidfilm }^{\circledast} / \\
\text { Ondissolve }^{T M}\end{array}$} & \multirow[t]{4}{*}{$\begin{array}{l}\text { Ondansetron } \\
\text { Hydrochloride }\end{array}$} & \multirow[t]{4}{*}{ Launched } & dispersible & & \multirow{4}{*}{$\begin{array}{l}{[20],} \\
75,[90, \\
91],[92 \\
, 93]\end{array}$} \\
\hline & & & & & & & dispersible & & \\
\hline & & & & & & & dispersible & & \\
\hline & & & & & & & dispersible & & \\
\hline & & WO-2011124570 & $\begin{array}{l}\text { APR Applied Pharma } \\
\text { Research SA; MonoSol Rx } \\
\text { LLC; tesa Labtec GmbH }\end{array}$ & $\begin{array}{l}\text { Zolmitriptan ODF } \\
\text { RapidFilm }^{\circledR}\end{array}$ & Zolmitriptan & Launched & dispersible & & $\begin{array}{l}{[94,} \\
95]\end{array}$ \\
\hline & & & & Aripiprazole ODF & Aripiprazole & $\begin{array}{l}\text { No Development } \\
\text { Reported }\end{array}$ & dispersible & & \\
\hline & & WO-2012110222 & $\begin{array}{l}\text { APR Applied Pharma } \\
\text { Research SA; tesa Labtec } \\
\text { GmbH }\end{array}$ & Olanzapine ODF & Olanzapine & Registered & dispersible & & [96] \\
\hline & & $\begin{array}{l}\text { WO-2009043588; } \\
\text { EP-02213278 }\end{array}$ & $\begin{array}{l}\text { APR Applied Pharma } \\
\text { Research SA; Ferrer } \\
\text { Internacional SA; tesa } \\
\text { Labtec GmbH }\end{array}$ & Donepezil ODF & Donepezil & Registered & dispersible & & [97] \\
\hline
\end{tabular}




\begin{tabular}{|c|c|c|c|c|c|c|c|c|c|}
\hline \multirow{2}{*}{$\begin{array}{l}\text { Brand name / } \\
\text { Designation }\end{array}$} & \multirow{2}{*}{$\begin{array}{l}\text { Owner / } \\
\text { Originator } \\
\text { Company }\end{array}$} & \multirow[t]{2}{*}{ Patent (s) } & \multirow{2}{*}{$\begin{array}{l}\text { Active Companies / } \\
\text { Partner / Distributor }\end{array}$} & \multirow[t]{2}{*}{ Products } & & & \multirow{2}{*}{$\begin{array}{l}\text { Oral Film } \\
\text { Type }\end{array}$} & \multirow[t]{2}{*}{ Polymer } & \multirow[t]{2}{*}{ Ref } \\
\hline & & & & & $\begin{array}{c}\text { Drug substance (if } \\
\text { any) }\end{array}$ & Phase / Status & & & \\
\hline \multirow[t]{6}{*}{ Schmelzfilm } & \multirow[t]{6}{*}{$\begin{array}{l}\text { Hexal } \\
\text { Pharmaceuticals }\end{array}$} & $\begin{array}{l}\text { WO- } \\
2007009801\end{array}$ & & $\begin{array}{l}\text { Olanzapin HEXAL }{ }^{\circledR} \text { SF } \\
\text { Schmelzfilm }\end{array}$ & Olanzapine & Launched & $\begin{array}{l}\text { orodispers } \\
\text { ible }\end{array}$ & \multirow{5}{*}{$\begin{array}{l}\text { Ethylcellulo } \\
\text { se } \\
\text { HPMC }\end{array}$} & \\
\hline & & $\begin{array}{l}\text { WO- } \\
2007009800\end{array}$ & & Anti-migraine & & * & & & \\
\hline & & $\begin{array}{l}\text { WO- } \\
2010115724\end{array}$ & & $\begin{array}{l}\text { Aripiprazole } \mathrm{HEXAL}^{\circledR} \mathrm{SF} \\
\text { Schmelzfilm }\end{array}$ & Aripiprazole & $*$ & $\begin{array}{l}\text { orodispers } \\
\text { ible }\end{array}$ & & \\
\hline & & & \multirow[t]{3}{*}{$\begin{array}{l}\text { Hexal } \\
\text { Sandoz }\end{array}$} & Risperidon $\mathrm{HEXAL}^{\circledast} \mathrm{SF}$ & Risperidon & Launched & $\begin{array}{l}\text { orodispers } \\
\text { ible }\end{array}$ & & \multirow[t]{3}{*}{ [98] } \\
\hline & & & & Donepezil-HCl Hexal SF & Donepezil & Launched & $\begin{array}{l}\text { orodispers } \\
\text { ible }\end{array}$ & & \\
\hline & & $\begin{array}{l}\text { WO- } \\
2012055947\end{array}$ & & $\begin{array}{l}\text { SildeHEXAL SF } \\
\text { (Tornetis) }\end{array}$ & Sildenafil & Launched & $\begin{array}{l}\text { orodispers } \\
\text { ible }\end{array}$ & \multirow{6}{*}{$\begin{array}{l}\text { Backing } \\
\text { layer - } \\
\text { HPC, HEC } \\
\text { Active layer } \\
\text { - } \\
\text { polycarbop } \\
\text { hil and } \\
\text { NaCMC }\end{array}$} & \\
\hline \multirow[t]{5}{*}{ BEMA $^{\mathrm{TM}}$} & \multirow{5}{*}{$\begin{array}{l}\text { BioDelivery } \\
\text { Sciences } \\
\text { International } \\
\text { (BDSI) }\end{array}$} & $\begin{array}{l}\text { WO- } \\
03086345 \\
\text { WO- }\end{array}$ & $\begin{array}{l}\text { KunWha Pharmaceutical } \\
\text { Co Ltd; Meda AB; TTY } \\
\text { Biopharm Co Ltd }\end{array}$ & Onsolis $^{\circledR}$ & Fentanyl & Launched & Buccal & & \multirow[t]{5}{*}{$\begin{array}{l}{[99-} \\
101]\end{array}$} \\
\hline & & $\begin{array}{l}\text { WO- } \\
\text { 2013096811; } \\
\text { WO- } \\
2010008863\end{array}$ & Endo Pharmaceuticals & BEMA $^{\circledast}$ Buprenorphine & Buprenorphine & Phase 3 clinical & Buccal & & \\
\hline & & & & BUNAVAIL ${ }^{\mathrm{TM}}$ & $\begin{array}{l}\text { Buprenorphine + } \\
\text { Naloxone }\end{array}$ & $\begin{array}{l}\text { NDA submitted to } \\
\text { FDA on July } 2013\end{array}$ & Buccal & & \\
\hline & & $\begin{array}{l}\text { WO20050163 } \\
21\end{array}$ & $\begin{array}{l}\text { BioDelivery Sciences } \\
\text { International Inc }\end{array}$ & BEMA $^{\circledast}$ Triptan & Triptan & Discovery & Buccal & & \\
\hline & & & Arius Pharmaceuticals Inc & BEMA $^{\oplus}$ Zolpidem & Zolpidem & $\begin{array}{l}\text { No Development } \\
\text { Reported }\end{array}$ & Buccal & & \\
\hline
\end{tabular}




\begin{tabular}{|c|c|c|c|c|c|c|c|c|c|}
\hline \multirow{2}{*}{$\begin{array}{l}\text { Brand name / } \\
\text { Designation }\end{array}$} & \multirow{2}{*}{$\begin{array}{l}\text { Owner / } \\
\text { Originator } \\
\text { Company }\end{array}$} & \multirow[t]{2}{*}{ Patent (s) } & \multirow{2}{*}{$\begin{array}{l}\text { Active Companies / } \\
\text { Partner / Distributor }\end{array}$} & \multirow[t]{2}{*}{ Products } & & & \multirow{2}{*}{$\begin{array}{l}\text { Oral Film } \\
\text { Type }\end{array}$} & \multirow[t]{2}{*}{ Polymer } & \multirow[t]{2}{*}{ Ref } \\
\hline & & & & & $\begin{array}{l}\text { Drug substance (if } \\
\text { any) }\end{array}$ & Phase / Status & & & \\
\hline $\begin{array}{l}\text { Fast } \\
\text { dissolving } \\
\text { Film }\end{array}$ & $\begin{array}{l}\text { Hughes } \\
\text { Medical Corp }\end{array}$ & & & & & & dispersible & & [20] \\
\hline \multirow[t]{4}{*}{$\begin{array}{l}\text { PharmaForm } \\
\text { technology }\end{array}$} & \multirow{4}{*}{$\begin{array}{l}\text { Auxilium } \\
\text { Pharmaceutic } \\
\text { als }\end{array}$} & WO-2010002418 & & Rotavax $^{\mathrm{TM}}$ & Rotavirus & $\begin{array}{l}\text { phase II clinical trials } \\
\text { (May 2013) }\end{array}$ & Buccal & & \multirow{4}{*}{$\begin{array}{l}{[50,51,} \\
103, \\
104]\end{array}$} \\
\hline & & & & & Testosterone & Discontinued & Buccal & & \\
\hline & & & & & Oxybutynin & $\begin{array}{l}\text { No Development } \\
\text { Reported }\end{array}$ & Buccal & & \\
\hline & & WO-2009151574 & & & Fentanyl & $\begin{array}{l}\text { No Development } \\
\text { Reported }\end{array}$ & Buccal & & \\
\hline Soluleaves ${ }^{\mathrm{TM}}$ & Bioprogress & $\begin{array}{l}\text { WO-2006114604 } \\
\text { (A3) }\end{array}$ & Meldex International & & Nicotine & $\begin{array}{l}\text { No Development } \\
\text { Reported }\end{array}$ & Buccal & $\begin{array}{l}\text { Cellulose } \\
\text { derivative }\end{array}$ & $\begin{array}{l}{[20,} \\
54]\end{array}$ \\
\hline \multirow{13}{*}{$\begin{array}{l}\text { Bio-FX Fast- } \\
\text { Onset ODF }\end{array}$} & \multirow[t]{13}{*}{ NAL Pharma } & \multirow[t]{13}{*}{ WO-2010062688 } & & & Selegiline & Discovery & \multirow[t]{13}{*}{ dispersible } & & \multirow{13}{*}{$\begin{array}{l}{[46,} \\
105]\end{array}$} \\
\hline & & & & & Rizatriptan Benzoate & Phase 1 Clinical & & & \\
\hline & & & & & Nicotine & Discovery & & & \\
\hline & & & & & Levocetirizine & Discovery & & & \\
\hline & & & & & Zolmitriptan & Discovery & & & \\
\hline & & & & & Sumatriptan & Discovery & & & \\
\hline & & & & & Sildenafil citrate & Phase 1 Clinical & & & \\
\hline & & & & & Tadalafil & Discovery & & & \\
\hline & & & & & Montelukast & Discovery & & & \\
\hline & & & & & Fentanyl & Discovery & & & \\
\hline & & & & & Cetirizine $\mathrm{HCl}$ & Discovery & & & \\
\hline & & & & & Donezepil & Discovery & & & \\
\hline & & & & & Zolmitriptan & Discovery & & & \\
\hline
\end{tabular}




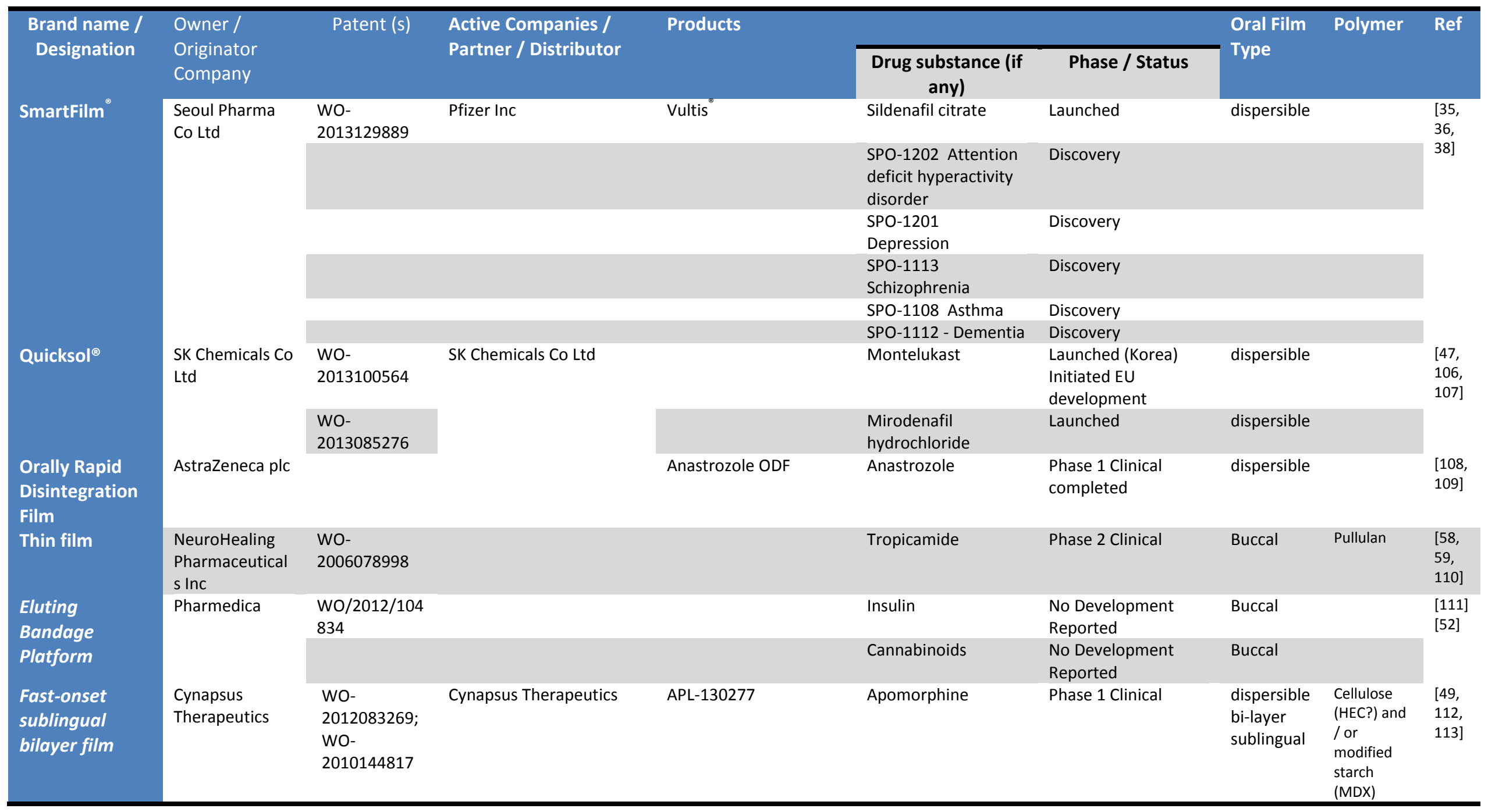




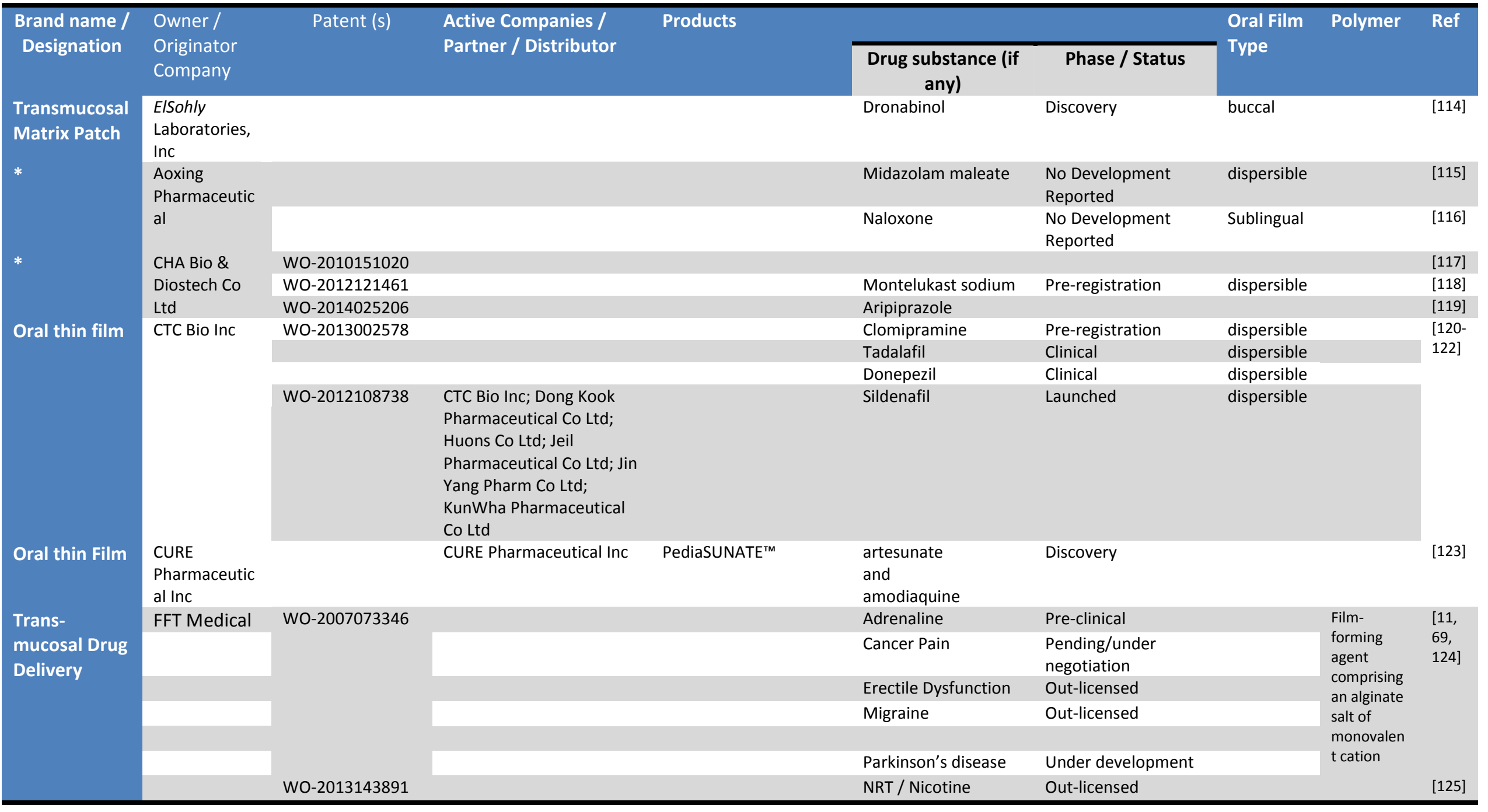




\begin{tabular}{|c|c|c|c|c|c|c|c|c|c|}
\hline \multirow{2}{*}{$\begin{array}{l}\text { Brand name / } \\
\text { Designation }\end{array}$} & \multirow{2}{*}{$\begin{array}{l}\text { Owner / } \\
\text { Originator } \\
\text { Company }\end{array}$} & \multirow[t]{2}{*}{ Patent (s) } & \multirow{2}{*}{$\begin{array}{l}\text { Active Companies / } \\
\text { Partner / Distributor }\end{array}$} & \multirow[t]{2}{*}{ Products } & & & \multirow{2}{*}{$\begin{array}{l}\text { Oral Film } \\
\text { Type }\end{array}$} & \multirow[t]{2}{*}{ Polymer } & \multirow[t]{2}{*}{ Ref } \\
\hline & & & & & $\begin{array}{c}\text { Drug substance (if } \\
\text { any) }\end{array}$ & Phase / Status & & & \\
\hline \multirow{13}{*}{$\begin{array}{l}\text { Oral } \\
\text { Dispersible } \\
\text { Film }\end{array}$} & Aavishkar & & & Tadalafil & Tadalafil & Launched & & & [126] \\
\hline & & & & Ondansetron $\mathrm{Hcl}$ & Ondansetron $\mathrm{Hcl}$ & Launched & & & [126] \\
\hline & & & & Simethicone & Simethicone & Launched & & & \\
\hline & & & & $\begin{array}{l}\text { Dextromethorphan } \mathrm{Hbr} \\
\text { Phenylephrine } \mathrm{Hcl} 2.5 \\
\text { mg Strips } \\
\text { (Cough \& Cold) }\end{array}$ & $\begin{array}{l}\text { Dextromethorphan } \\
\mathrm{Hbr} \\
\text { Phenylephrine } \mathrm{Hcl} \\
2.5 \mathrm{mg}\end{array}$ & Launched & & & \\
\hline & & & & Vitamin B12 strips & Vitamin B12 & Launched & & & \\
\hline & & & & Vitamin D3 strips & Vitamin D3 & Launched & & & \\
\hline & & & & Electrolyte Strips & $\begin{array}{l}\text { Vitamin } \mathrm{B} 12+ \\
\text { Vitamin } \mathrm{C}+\text { Sodium }+ \\
\text { Potassium }\end{array}$ & Launched & & & \\
\hline & & & & Energy strips & $\begin{array}{l}\text { Caffeine + Vitamin } \\
\text { B12 + Vitamin E + } \\
\text { Vitamin B6+ Biotin+ } \\
\text { Vitamin B5 }\end{array}$ & Launched & & & \\
\hline & & & & Melatonin Strips & Melatonin & Launched & & & \\
\hline & & & & Teeth Whitening Strips & $\begin{array}{l}6 \% \text { Hydrogen } \\
\text { Peroxide }\end{array}$ & Launched & & & \\
\hline & & & & $\begin{array}{l}\text { Breath Freshening } \\
\text { Strips }\end{array}$ & & Launched & & & \\
\hline & & & & & AVISH-01 ( AIDS) & Discovery & & & [11] \\
\hline & & & & & $\begin{array}{l}\text { AVISH-02 (Vaginal } \\
\text { infections) }\end{array}$ & R\&D completed & & & \\
\hline
\end{tabular}




\section{Market overview}

The feverous need of launching on the market renewed drug delivery systems of already approved drugs, in order to avoid generic competition, allied with the increased patient's compliance concern, have driven the attention of the industry for the orodispersible technology. There are enormous costs and time consumption in the discovery and development of new chemical entities and at the same time there is a real need to improve the efficacy, safety and compliance of some marketed products [127]. In fact, almost a quarter of the drugs in the market do not provide the expected commercial returns due to its poor bioavailability and undesirable pharmacokinetics, demanding the development of innovative, better and suitable drug delivery systems [128]. Therefore, the field of novel drug delivery technologies is highly competitive, but also very rewarding. The average cost of a new formulation is considerable lower than the cost involving the development of a new chemical entity, in about 40 million dollars, and generally takes also less time to develop (approximately 4 to 5 years). Actually, the changing market trends are very clear, the FDA approvals revealed a majority of reformulations or combinations of current approved products, in contrast with the $25 \%$ of new drugs approvals. In 2012, the total drug delivery market (DD) worth $\$ 142.5$ billion [4], and presently, for the 10 most-popular DD technologies, its estimated market value of $\$ 81.5$ billion [129] and is expected to achieve $\$ 92$ billion by 2016 [130].

The oral formulations are the number-one segment of the drug delivery, retaining more the larger sliver of the global market share [129]. The orodispersible drug delivery systems have found its space as mainstream pharmaceutical products. In fact, eight in ten patients prefer orodispersible dosage forms over the traditional solid oral dosages. Orodispersible films initially emerged as an option for rapid drug delivery, and later with the buccal films as an alternative for low bioavailability drugs. From the market standpoint, oral films were immediately appointed as a successful delivery system probably fueled by the huge success of Listerine ${ }^{\circledR}$ Pocket Packs $^{\circledR}$. Actually, oral films proved to be a rewarding commercial platform with a growing rate of 500 million dollars by year since 2006 and reached the 2 billion dollars in 2010 [20]. Recent market analyses revealed that the oral film manufacturers have had rapid revenue growth from 2009-2014 and a similar trend is expected at least until 2019. It was estimated an annual growth of $17,1 \%$ in the same period for this sector [18]

In 2001, Pfizer launched the Listerine ${ }^{\circledR}$ Pocket Packs ${ }^{\circledR}$ in the market, the first commercial oral film and a blockbuster that in one year exceeded 175 million dollars [9]. Nowadays, under new ownership, it still remains a viable business, although it is a shadow of its initial success. In 2003, Chloraseptic ${ }^{\circledR}$ Relief Strips, the first drug substance loaded oral thin film reached the market [131]. Chloraseptic ${ }^{\circledR}$ Relief Strips with benzocaine provided an immediate and convenient relief of sore throat pain. In 2004, Novartis debuts in the oral film market with Triaminic $^{\circledast}$ Thin Strips and Theraflu ${ }^{\circledR}$ Thin Strips to treat the most common symptoms of a cold in young children (ages 6-12) and older children (> 12 year old) or adults, respectively. These products were considered the "Best Product of 2004" only three weeks after their official launch, however, they are no longer available in the shelves [132]. The melts-in-mouth portable delivery platform, with an easy and pleasant administration for children was probably the first multi-symptom cough and cold medicines that provided a fast and accurate dosing. In the beginning of 2012, Novartis decided to discontinue the manufacturing and distribution of Children's Triaminic ${ }^{\circledR}$ Thin Strips "after a careful consideration" [133]. Novartis claimed that this decision was based upon a business need, but there are some suggestions that the removal of these products included production issues and poor sales $[134,135]$. The latter may probably be a reflection of the decline in the consumption of cough and cold medications due to the implementation of restrictions in 2007 [136, 137].

Nevertheless, Novartis and Pfizer as marketing partners remain as top players in the thin film industry, their capitalization in these success brand products allowed them to gain a strong position in the field [138]. The thin film industry exhibits a high level of competition, but until 2010 no prescription oral film product had reached the market. It was a slow ride from the over-the-counter (OTC) market until the first prescription has been approved. 
It was only in 2010 that the first Rx oral film, the ondansetron Rapidfilm ${ }^{\circledR}$ (Setofilm ${ }^{\circledR}$ ) and the ondansetron Pharmfilm ${ }^{\circledR}$ (Zuplenz ${ }^{\circledast}$ ), received European and FDA approval, respectively. Labtec GmBh, APR Applied Pharma Research SA (APR) and MonoSol Rx LLC (MonoSol) entered in the market with an ondansetron oral thin film for the prevention of nausea and vomiting, staunch that would capture a broad share of an appellative market that generated 1.9 billion dollars in the same year [139]. A month after Zuplenz ${ }^{\circledR}$ launch, MonoSol together with Reckitt Benckiser Pharmaceuticals received FDA approval for Suboxone ${ }^{\circledR}$ sublingual film $[135,140]$. This thin film, with two drug substances, buprenorphine and naloxone, approved for the treatment of opioid dependence in adults, was a huge success contributing to boost the oral films market. In 2011, Suboxone ${ }^{\circledR}$ thin film recorded sales of 513 million dollars and accounted for 96 percent of the oral transmucosal film market [141]. In 2012, US sales of Suboxone ${ }^{\circledR}$ sublingual film alone exceeded 1.5 billion dollars and continue with gradual growth [142]. During clinical trial the patients seemed to prefer the Suboxone ${ }^{\circledR}$ sublingual film rather than Suboxone ${ }^{\circledR}$ sublingual tablets, due to its fast dissolution and more pleasant taste profile [140]. These factors attracted other companies, as Alvogen Pine Brook, Actavis, Intelgenx and BioDelivery Sciences International (BDSI) to develop similar technologies. In fact, the first three companies have recently filled abbreviated new drug applications (ANDA) for generic products of Suboxone ${ }^{\circledR}$ sublingual film whereas BDSI submitted a NDA with its own buccal film technology, the BUNAVAIL ${ }^{\mathrm{TM}}$. BDSI believes that BUNAVAIL ${ }^{\mathrm{TM}}$, which adheres to the inside of the cheek, has the potential to offer advantages over Suboxone ${ }^{\circledR}$ sublingual film [43, 142].

At the moment, none of these competitors' products are on the market since a patent infringement lawsuit against these applicants was submitted by Reckitt Benckiser [143]. The success of the Reckitt Benckiser's prescription thin film proved the viability and value of this pharmaceutical form in the Rx market. In the US the oral films had come into a strong prominence and the prescriptions confirm the preference of these pharmaceutical forms [144].

\section{Market outlook}

It is evident the increasing consumer acceptance of thin films and the market success that can be achieved. Generally, for this dosage form the OTC market is the preferred due to the close proximity with the consumer and the easily advertising. Additionally, in the USA market the convenience store category is extremely important and it has a high sales impact. There are more than 140,000 stores across the US, which achieved a gross sale of 708.2 billion dollars in 2012, with a continuous sale grew every year. Regarding the OTC products, in the Convenience Store News 2013 Industry Report, they are included as health and beauty care products, which in overall had an average gross margin of about 46.89 percent in 2012 [20, 145]. It is a very tempting market that may lead to precipitated launches that sometimes may fail the expectations. As example of that, the Sudafed PE ${ }^{\mathrm{TM}}$ Quick Dissolve Strips was launched by Pfizer in the middle of 2005, for the relief of sinus pressure and congestion [146, 147]. The brand sales were probably lower than expected due to the intense competition in the cough-and-cold products segment. In fact, considering only Triaminic ${ }^{\circledR}$ and Theraflu ${ }^{\circledR}$, launched two years earlier, Sudafed $\mathrm{PE}^{\mathrm{TM}}$ effectively did not leverage a strong point-of-difference, not even in the price (Sudafed $\mathrm{PE}^{\mathrm{TM}}$ approximately $0,45 \$$ /film and Triaminic approximately $\left.0,5 \$ / \mathrm{film}\right)[132,148]$. In addition, it should be mentioned also that this segment sale were also off due to the implementation of some constraint measures for cough-and-cold medicines administration.

Nevertheless, all this background experiences are important for planning and develop new oral film. It is crucial to consider that the market success of a new oral film depends on its capacity of differentiate from its competitive set, beyond an attractive new dosage form. A product can achieve a decisive point of difference by its unique characteristics which may engage the customer's attention and bring also some significance. Therefore, considering the Rx market, it would be wise to deliver innovative drug substances, or others that bring additional value, to the oral film platform, otherwise it will be difficult to achieve success. 
The manufacturing process of the oral films, when comparing with some complex oral delivery systems, is costeffective and generally results in affordable end-products [20]. However, the majority of the oral films is more expensive than conventional oral dosage forms and may not represent a clear benefit for the consumer. It should be reminded that once the form novelty wears off, it must be found a way to gain advantage over the other competitors / brands that do equal efforts for offering equivalent products.

On the other hand, considering the previous discussion, there is still a poorly explored market with this delivery platform, the veterinary market. It is well known that in general the animals tend to reject, spit out or vomit conventional dosage forms. In fact, giving oral medications to pets can be sometimes a dangerous practice, which can also reduce even the owner compliance, and compromise the treatment. It was evaluated the overall owner-perceived acceptability and the easiness administration of an orodispersible film compared with gelatin capsules in cats. Although there were no significant differences in the general acceptability of the owners, it was shown that the orodispersible films facilitated significantly the administration of the medication [149, 150]. Currently, few companies have developed oral films applied to this segment, and the available products are scarce and limited to OTC products based on blends of herbal extracts [151, 152]. Additionally, IntelGenx presents in its pipeline the VetaFilm which rapidly hydrate and adheres upon contact with the tongue. It cannot be spit out and offers appealing flavors and scents to increase the acceptability in pets [31].

The veterinary industry includes several health products such as biologicals, medicated feed additives and veterinary pharmaceuticals that deals with a wide range of products: metabolic drugs, anti-infectives, reproductive aids, feed additives, vaccines, imaging diagnostics, topical solutions, parasite controls, oncologic, cardiovascular, and osteoarthritis drugs [153].

It is estimated that the global animal health care market is worth between 92 and 102 billion dollars [154]. Regarding pets, there is an evident trend of the owners spending more money each year on their pets' health. In the US, it is expected that pet-owners spending will reach 33 billion dollars in 2014. This market segment will always be far from recession, driven by the idea that pets are considered family members. Besides that, the farm animals' health has been in the spotlight as a result of growing awareness of the impact that animal health may have in the human food safety and public health. The current unmet animal health needs, especially in animal disease surveillance, vaccines and lack of drugs in the senior animal veterinary care market (oncology, cognitive dysfunction syndrome, antidepressants) may also fuel the veterinary market growth [153]. Although, US remain the largest regional market for animal medication, Asia-Pacific is the fastest growing regional market. Regarding the products segment, the nutritional chemicals and parasiticides have the majority share in the total dollar sale, whereas vaccines and diagnostics are the fastest growing products segments [155, 156].

It is clear that animal health is far to be little and insignificant health care niche. Actually, keeping pets and farm animals healthy might be a huge business. Therefore, innovative delivery platforms such as the orodispersible films, that quickly dissolve and become sticky in contact with saliva, can easily became valued by pet owners and animal caregivers.

Another unexplored market with these technology platforms is the biologics products. There are some references and efforts in the development of these type of products especially in immunotherapy, but at the moment no product had already reached the market. For example, vaccines in oral film formulations may have significant advantages. Despite of the needle free delivery, which may increase patient compliance, the higher stability may simplify the worldwide storage and distribution. The simplification of the logistic would also favor the mass immunization campaigns $[157,158]$. In fact, this type of small and stable vaccines could be easily sent by mail. Furthermore, it is expected that the human vaccine industry forecast reaches 41.85 billion dollars by 2018 [159], with a CARG growth of 62.81 percent from 2013 to 2018 [160].

In addition to the quick-dissolving oral film influenza vaccine, under development by BiondVax - MonoSol Rx, others had already reported the development of oral film vaccines. During 2007, Johns Hopkins students had reported the development of an oral film vaccine, the rotavirus thin film delivery system which may surpass the drawbacks of the liquid vaccine. The original idea came from Aridis Pharmaceuticals that owns the rotavirus vaccine stable at room temperature and gave the challenge to the researchers from the Johns Hopkins School of 
Medicine. The oral film system is composed by a FDA-approved biocompatible polymer, which is pH-responsive. This composition design avoids the degradation of the vaccine in the stomach acid, allowing its release in the small intestine, triggering the immune response. According to their patent application these $\mathrm{pH}$-sensitive microparticles are composed by a complex polymeric mixture, a copolymer of methacrylic acid or acrylic acid, as an Eudragit ${ }^{\circledR}$ - like copolymer, a pluronic polymer, a chitosan or a derivative or a combination thereof; and possible additional components as a surfactant, a sugar, a buffering salt and or a combination thereof [104]. The Eudragit ${ }^{\circledR}$ polymers are an Evonik Industries AG trademark that offer a full flexibility of $\mathrm{pH}$-dependent drug release [161]. In fact, the manufacture method to prepare these microparticles includes the blend of two different grades of Eudragit ${ }^{\circledR}$, possibly to accurately define the $\mathrm{pH}$ release of the drug. An anionic copolymer of methacrylic acid with ethyl acrylate is balanced with an anionic copolymer of methacrylic acid with methyl methacrylate, so the drug release $\mathrm{pH}$ may be achieved somewhere between 5.5 and 7 . The oral film composition is based on water-soluble polymers, as PVP or PVA, mucoadhesive polymers, as PEO and / or sodium alginate.

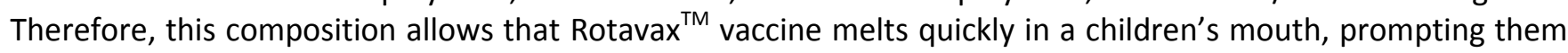
to swallow it. Unfortunately, no additional developments had been reported since then [20, 162]. Currently, the Johns Hopkins School of Medicine also has a clinical trial ongoing to evaluate the Safety and Efficacy of a sublingual dissolving film for Peanut Allergy. The estimated date for the completion of the study is in the beginning of 2015 and it is expected that this sublingual immunotherapy with a dissolving peanut extract film has the potential to improve the efficacy of this type of treatment $[163,164]$.

It has been an increasing interest in the development of micro- and / or nano- drug delivery systems associated to buccal polymeric matrices for a transmucosal delivery [165-171]. These nanoparticle systems for oral absorption have been recently explored to incorporate poorly soluble drugs, to extend and improve the buccal release, to provide an improvement in the drug targeting and also to increase drug stability. These systems generally include nanosuspensions [166, 167, 169], nanofibrous matrix system [168], solid lipid nanoparticles[165, 171], PEG-b-PLA nanoparticles [171], submicron and nanosized particles of lysozyme (Lys)loaded d , I-valine (Val) [170]. The majority of these studies were initially applied to the proteins, especially due to its large size, low oral bioavaibility and poor stability, but they are currently extended to non-biologic drugs. Nevertheless, for the development of this type of systems it is important to consider the size of the coating particles. The size and shape of the particles may lead to undesirable aggregation and loss in the homogeneity, which could compromise the physical stability of the films in terms of both mechanical and mucoadhesive properties.

\section{Conclusion}

The main catalysts of the drug-delivery market are the patent cliff and more informed and autonomous consumers [4]. Therefore, the demand side for pharmaceutical treatments has been changing and nowadays the approach is more patient - centered and quality- based.

Recent reports refer that in the next five-year period, many oral film drug producers will focus on extend their drug pipeline through other therapeutic classes [18]. At the same time it is expected that the formulation complexity may contribute to technical, manufacturing and regulatory barriers that may lessen the growth of the oral film market [4]. So, it is critical that the value added by any new delivery platform boosts its own growth by the continuous improvement of consumer compliance and by enticing new consumers. 


\section{Acknowledgements}

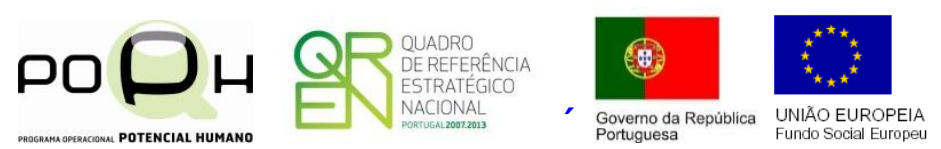

Funded by FCT grant, reference SFRH / BDE / 51271 / 2010 and by QREN SI-I\&DT No.18472 


\section{References}

[1] H. Rosen, T. Abribat, The rise and rise of drug delivery, Nature reviews. Drug discovery, 4 (2005) 381-385.

[2] G. Research, Drug delivery market to reach $\$ 199 b n$ by 2016 , says GBI Research, in, GBI Research, http://www.in-pharmatechnologist.com/Materials-Formulation/Drug-delivery-market-to-reach-199bn-by-2016says-GBI-Research, 2010.

[3] R. Report, Innovative Drug Delivery Systems: Novel Product and Formulation Technology Licensing Opportunities for Generic Pharmaceutical Companies, in, http://www.prweb.com/releases/innovative-drugdelivery/systems-market-analysis/prweb11170481.htm, 2013.

[4] MarketsandMarkets, Drug Delivery Technologies Market (Metered Dose Inhalers, Needle-Free Injectors, Auto-Injectors, Nasal Sprays, Transdermal Patches, Nebulizers, Infusion Pumps, Drug Eluting Stents, Sustained Release, Ocular Implants) - Global Forecasts To 2017 in, marketsandmarkets.com, http://www.marketsandmarkets.com/Market-Reports/drug-delivery-technologies-market-1085.html, April 2013.

[5] J.T. Schiele, R. Quinzler, H.D. Klimm, M.G. Pruszydlo, W.E. Haefeli, Difficulties swallowing solid oral dosage forms in a general practice population: prevalence, causes, and relationship to dosage forms, European journal of clinical pharmacology, 69 (2013) 937-948.

[6] S. Stegemann, M. Gosch, J. Breitkreutz, Swallowing dysfunction and dysphagia is an unrecognized challenge for oral drug therapy, Int J Pharm, 430 (2012) 197-206.

[7] R. Bala, P. Pawar, S. Khanna, S. Arora, Orally dissolving strips: A new approach to oral drug delivery system, International journal of pharmaceutical investigation, 3 (2013) 67-76.

[8] E.M. Hoffmann, A. Breitenbach, J. Breitkreutz, Advances in orodispersible films for drug delivery, Expert opinion on drug delivery, 8 (2011) 299-316.

[9] B. Levinson, The Tale Of TiVo And Why Great Brands Fall From Grace in: Leadership, http://www.fastcompany.com/3002032/tale-tivo-and-why-great-brands-fall-grace, 2012.

[10] Evalueserve, Mouth Dissolving Films - Landscape Study, in, Bluepharma confidential information, 2011.

[11] R. Analysis, Oral Thin Films - Market, 2015 - 2025, in, 2015.

[12] A. Breitenbach, S. Braun, R. Schliephacke, Orodispersible films for the manufacturing of individualised medicine or for large scale production, in, Google Patents, 2013.

[13] E.M. Janssen, R. Schliephacke, A. Breitenbach, J. Breitkreutz, Drug-printing by flexographic printing technology--a new manufacturing process for orodispersible films, Int J Pharm, 441 (2013) 818-825.

[14] FierceDrugDelivery, Oral insulin film from MonoSol and Midatech, in: FierceDrugDelivery, http://www.fiercedrugdelivery.com/special-reports/top-5-insulin-delivery-techs-watch/3-oral-insulin-filmmonosol-and-midatech, 2012.

[15] N.J. Warren, MonoSol Rx and BiondVax Collaborate to Develop Oral Film Delivery of Flu Vaccine, in: G. NEWSWIRE (Ed.), http://globenewswire.com/news-release/2012/11/07/503159/10011500/en/MonoSol-Rxand-BiondVax-Collaborate-to-Develop-Oral-Film-Delivery-of-Flu-Vaccine.html, 2012.

[16] B. Pulliam, Oral thin film vaccine preparation, in, Google Patents, 2012.

[17] S. Cohen, E. Ron, Y. Rubin, New oral dissolving films for insulin administration, for treating diabetes, in, Google Patents, 2012.

[18] IBISWorld, Thin Film Drug Manufacturing in the US: Market Research Report, in, http://www.ibisworld.com/industry/thin-film-drug-manufacturing.html, 2015.

[19] J.O. Morales, J.T. McConville, Manufacture and characterization of mucoadhesive buccal films, European journal of pharmaceutics and biopharmaceutics : official journal of Arbeitsgemeinschaft fur Pharmazeutische Verfahrenstechnik e.V, 77 (2011) 187-199.

[20] R.P. Dixit, S.P. Puthli, Oral strip technology: overview and future potential, Journal of controlled release : official journal of the Controlled Release Society, 139 (2009) 94-107. 
[21] P. Newswire, MonoSol Rx Secures 505(b)(2) Regulatory Pathway for Montelukast Sodium Oral Soluble Film, in: Pharmaceuticals, http://www.prnewswire.com/news-releases/monosol-rx-secures-505b2-regulatorypathway-for-montelukast-sodium-oral-soluble-film-118904699.html, 2011.

[22] N. OMX, MonoSol Rx Developing Migraine Product for Treatment of Pain and Nausea in a Single Dose, in: Globe newswire, http://globenewswire.com/news-release/2012/09/13/490573/10005040/en/MonoSol-RxDeveloping-Migraine-Product-for-Treatment-of-Pain-and-Nausea-in-a-Single-Dose.html, 2013.

[23] M. Rx, MonoSol Rx Completes Thin Film Escitalopram Oxalate Pilot in: Product Development, http://www.monosolrx.com/content/media/releases/news 80408.html, 2008.

[24] Reuters, Company Overview - Full description, in: Pharmaceuticals-Generic / Specialty, http://in.reuters.com/finance/stocks/companyProfile?symbol=MR1.BE.

[25] M. Rx, Development pipeline, in, http://www.monosolrx.com/content/pipeline/overview.htm, 2013.

[26] P. Newswire, Midatech Raises $f 10$ Million to Advance Gold-nanoparticle Technology for Diabetes and Cancer Treatment, in, http://www.prnewswire.com/news-releases/midatech-raises-10-million-to-advance-goldnanoparticle-technology-for-diabetes-and-cancer-treatment-230611141.html, 2013.

[27] Reuters, Biondvax Pharmaceuticals Ltd Signs New Collaboration Agreement With MonoSol Rx, in, http://in.reuters.com/finance/stocks/BNDX.TA/key-developments/article/2625222, 2012.

[28] B.A. LEICHS C, LEHRKE I, GALFETTI P, in, WO, 2008.

[29] t.L. GmbH, Rapidfilm ${ }^{\circledR}$ Oral film technology: an innovative form of oral medication in, http://www.tesalabtec.com/eng/rapidfilm/overview/oral-film-technology-an-innovative-form-of-oral-medication,4353970.html.

[30] A. Pharma, Pharmaceutical Global Development, in, http://www.apr.ch/site/licence/index.htm\#/site/licence/pharma.htm.

[31] I. Corp., Pipeline, in, http://www.intelgenx.com/pipeline/, 2006.

[32] I.C.R.B. Ltd., IntelGenx and RedHill Biopharma Receive Complete Response Letter From FDA for VersaFilm(TM) Oral Film Product for Acute Migraines in, http://ir.redhillbio.com/releasedetail.cfm?ReleaselD=823064, 2014.

[33] L. KyuKyu Pharmaceuticals Co., QQ's creativity is always focused around "Q". in, http://www.qqp.co.jp/english/product/film.html.

[34] P.M.B. Intelligence, Nippon Kayaku Cancer Pain Relief Drug To Enter Phase II Development, in: Pharm Asia News, Elsevier Business Intelligence, http://www.elsevierbi.com/publications/pharmasianews/2009/7/15/nippon-kayaku-cancer-pain-relief-drug-to-enter-phase-ii-development, 2009.

[35] T.R. Cortellis, LG Life Sciences' Zemiglo granted the Korea New Drug Award, Pharma Koreana, 201323 (April 01) 25, Source ID: 1447789 in: Development Profile, 2013.

[36] T.R. Cortellis, High-dose unit film formulations expected to heat the erectile dysfunction drug market, Pharma Koreana, 201323 (January 01), Source ID: 1357733 in: Development Profile, 2013.

[37] S. Pharma, R\&D Status, in, Seoul Pharma, http://www.seoulpharma.com/eng/sub2 02.html, 2012.

[38] H.S.J. Kim, Hyun Jun; Oh Dong Hoon; Park, Jin Gyu; Kim Hyun Soo; Um Jin Hee; Lee, Jin Hoo; Lim Yeon Jin; Jang Ik Hyeon; Um, Jin Hee; Kim, Dal Geun; Chang, Ik Hyeon; Yeon, Kyu Jeong; Jung, Kyung Tae; Park Jin Gyu; Yeon Kyu Jeong; Jeong Hyun Jun High-content fast dissolving film with masking of bitter taste comprising sildenafil as active ingredient, in, Seoul Pharma. Co., Ltd. , 2013.

[39] I. BioDelivery Sciences International, BEMA ${ }^{\circledR}$ Technology, in, http://www.bdsi.com/BEMA Technology.aspx, 2014.

[40] H. Business, BioDelivery Sciences and Meda Launch ONSOLIS fentanyl buccal soluble film, in: Cancer Weekly, http://business.highbeam.com/408962/article-1G1-209729052/biodelivery-sciences-and-meda-launchonsolis-fentanyl, 2009.

[41] A. Pešić, Manufacturing of ONSOLIS is all go, says BioDelivery Sciences, in: Contract Manufacturing, Outsourcing-pharma.com, http://www.outsourcing-pharma.com/Contract-Manufacturing/Manufacturing-ofONSOLIS-is-all-go-says-BioDelivery-Sciences, 2010. 
[42] PRWeb, FDA delays the re-launch of Onsolis (Fentanyl buccal soluble film): AttorneyOne.com Warns the Threat from Fentanyl Severe Adverse Events Remains, in, http://www.prweb.com/releases/2012/3/prweb9294813.htm, 2012.

[43] P. Newswire, BioDelivery Sciences Provides an Update of Anticipated 2014 Milestones, in: Clinical Trials \& Medical Discoveries, http://www.prnewswire.com/news-releases/biodelivery-sciences-provides-an-update-ofanticipated-2014-milestones-239591691.html, 2014.

[44] B. Wire, BioDelivery Sciences and Meda Announce European Approval of BEMA Fentanyl (BREAKYL), in, http://www.businesswire.com/news/home/20101020005551/en/BioDelivery-Sciences-Meda-Announce-

European-Approval-BEMA\#.Uv36r3cacbE, 2010.

[45] DataPharm, Breakyl 400mcg buccal film, in, https://www.medicines.org.uk/emc/medicine/28360.

[46] N. Pharma, Bio-FX Fast-Onset Oral-Cavity ODF, in: O. technology (Ed.), http://nalpharma.com/ourtechnology/bio-fx/, 2014.

[47] S. CHEMICALS, R\&D Life Science, in, SK CHEMICALS, http://www.skchemicals.com/en/sub1-3-3-4.asp.

[48] S. Chemicals, SK chemicals Sustainability Report 2012, in: Healthcare and Earthcare, http://www.skchemicals.com/korean/main/img/2012 eng.pdf, 2012.

[49] Cynapsus, APL-130277 Overview, in: APL-130277 Product, http://www.cynapsus.ca/general.php?pid=7, 2014.

[50] I. Auxilium Pharmaceuticals, Auxilium Pharmaceuticals Announces Top-Line Results for Phase II Androgen Replacement Transmucosal Film Proof of Concept Study in, http://www.sec.gov/Archives/edgar/data/1182129/000119312505153257/dex991.htm, 2005.

[51] I. Auxilium Pharmaceuticals, Auxilium Pharmaceuticals Announces Licensing Agreement with PharmaForm for Transmucosal Film Technology, in, http://ir.auxilium.com/phoenix.zhtml?c=142125\&p=irolnewsArticle2\&ID=671741\&highlight $=$.

[52] P. Ltd., Technology, in, http://www.pharmedica.co.il/pipeline.asp, 2014.

[53] A.C. Arun Arya, Vijay Sharma and Kamla Pathak, Fast Dissolving Oral Films: An Innovative Drug Delivery System and Dosage Form, PharmTech, 2 (2010) 576-583.

[54] N.E. Zbygniew, S.A. John, Water-soluble films comprising nicotine, in, Google Patents, 2006.

[55] K. Megget, New oral film takes the heat out of manufacturing, in, http://www.inpharmatechnologist.com/Ingredients/New-oral-film-takes-the-heat-out-of-manufacturing, 2007.

[56] P.L. INC., Paladin Submits Patent Application for Thinsol(TM), a Novel Oral Ingestible Film Composition Delivery System in, Marketwired, http://paladin-labs.mwnewsroom.com/manual-releases/2007/PaladinSubmits-Patent-Application-for-Thinsol\%28TM\%29, 2007.

[57] O. inc., Products-Technology description, in, http://www.odfpharma.com/index.php?option=com content\&view=article\&id=62\&ltemid=54\&lang=en, 2011.

[58] N. Pharmaceuticals, NH004 Program Summary, in, http://www.neurohealing.com/NH004ProductSummary-2012t.pdf, 2012.

[59] N.P. Inc., Study of the Safety and Efficacy of Tropicamide Thin Films to Reduce Hypersalivation in Parkinson's Patients, in, Clinicaltrials.gov, http://clinicaltrials.gov/show/NCT01844648, April 29, 2013.

[60] P.Z. online, Orale Filme - Auf die Zunge, fertig, los, in: Pharmazie, http://www.pharmazeutischezeitung.de/index.php?id=35328, 2010.

[61] L.L.T.-S. AG, Oral thin films - a new evolution step for active substances, in, http://www.Itslohmann.de/en/innovation/orale-wirkstoff-filme.html, 2014.

[62] M.a.H.p.R.A.M.r.M.a.M. Devices, Sidenafil Sandoz 25mg, 50mg and $75 \mathrm{mg}$ orodispersible films, in: P.A. report (Ed.) Decentralised Procedure, http://www.mhra.gov.uk/home/groups/par/documents/websiteresources/con303960.pdf, 2013.

[63] H.-H.o.M. Agencies, Olanzapin HEXAL SF $5 \mathrm{mg}$ Schmelzfilm, in: MR Number: DE/H/1721/001 http://mri.medagencies.org/Human/Product/Details/8994, 2014. 
[64] K. Klokkers, T. Kohr, K.T. Kramer, P. Obermeier, Oral, quickly disintegrating film, which cannot be spit out, for a neuroleptic drug, in, Google Patents, 2007.

[65] M. Medicines and Healthcare products Regulatory Agency, PAR Donepezil $5 \mathrm{mg}$ and $10 \mathrm{mg}$ Orodispersible Films PL 04416/1213-8, Donepezil 5 mg Orodispersible Films in: P.A. Report (Ed.) UK/H/2519-21/001-2/DC http://www.mhra.gov.uk/home/groups/par/documents/websiteresources/con132093.pdf.

[66] M. Regulating Medicines and Medical devices, Sildenafil Sandoz $25 \mathrm{mg}, 50 \mathrm{mg}$ and $75 \mathrm{mg}$ orodispersible film, in: Public Assessment Report, http://www.mhra.gov.uk/home/groups/par/documents/websiteresources/con303959.pdf, 2013.

[67] A. Krekeler, D. Neumann, Preparation of orodispersible films, in, Google Patents, 2012.

[68] P. Newswire, Nutra3 Complex(R) Develops New Oral Strips - Radiant \& Ageless Beauty From Within!, in, http://www.prnewswire.com/news-releases/nutra3-complexr-develops-new-oral-strips---radiant--ageless-

beauty-from-withintm-90744224.html, 2010.

[69] F. Medical, Trans-mucosal Drug Delivery, in, FFT Medical, http://fftmedical.se/, 2015.

[70] Susan Banbury, K. MacGregor, Pediatric Delivery, in: Drug Delivery Technology, 2011, pp. 34-40.

[71] S.-H.S.L. Leung, Robert S.; Kulkarni, Neema; Kumar, Lori D.; Spence Leung, Sau-Hung; Sorg, Albert F.; Kumar, Lori Dee; Sorg, Albert Fast dissolving orally consumable films, in, Mcneil-Ppc, Inc. , 2001.

[72] N.A. Paiement, Angela; Zerbe, Horst; Long, Cormac Solid oral film dosage forms and methods for making same, in, IntelGenx Corporation 2011.

[73] N.N. Nadeau Genevieve; Fortier, Geneviève; Belanger Eve; Fortier Nicole; Bélanger, Eve Ingestible film composition, in, Squire Pharmaceuticals Inc. , 2009.

[74] R.K. Yang, R.C. Fuisz, G.L. Myers, J.M. Fuisz, Thin film with non-self-aggregating uniform heterogeneity and drug delivery systems made therefrom, in, Google Patents, 2004.

[75] R.K. Yang, R.C. Fuisz, G.L. Myers, J.M. Fuisz, Polyethylene oxide-based films and drug delivery systems made therefrom, in, Google Patents, 2006.

[76] R.K. Yang, R.C. Fuisz, Systemes d'apport sous forme de film a base de glucane, in, Google Patents, 2003.

[77] B.A. Bogue, K. Davidson, L. Miloshoff, G.L. Myers, G. Slominski, Method and system for forming a pharmaceutical product directly onto a packaging surface, in, Google Patents, 2012.

[78] R.C. Fuisz, J.M. Fuisz, Biocompatible film with variable cross-sectional properties, in, Google Patents, 2012.

[79] E. Dadey, A.M. Schobel, Steroid hormone delivery systems and methods of preparing the same, in, Google Patents, 2013.

[80] T. Mickle, Homoarginine prodrugs and/or conjugates of amphetamine and other stimulants and processes for making and using the same, in, Google Patents, 2013.

[81] T.C. Mickle, Polar hydrophilic prodrugs of amphetamine and other stimulants and processes for making and using the same, in, Google Patents, 2008.

[82] B.A. Bogue, B.J. Boone, M. Hariharan, S.D. Hilbert, G.L. Myers, P. Sanghvi, Sublingual and buccal film compositions, in, Google Patents, 2012.

[83] A. Pharma, $2.5 \mathrm{mg}$ and $5 \mathrm{mg}$ Zolmitriptan ODF, in, http://www.apr.ch/site/licence/index.htm\#/site/licence/p pain zolmitriptan rf.htm.

[84] A. Ishise, 石瀬章浩, H. Nishikawa, 西川久信, Intraorally dissolving film preparation, in, Google Patents, 2013.

[85] T. Awamura, 粟村努， H. Nishikawa，西川久信， T. Inagi, Film preparation containing loperamide hydrochloride, in, Google Patents, 2010.

[86] K. Furusawa, Process for producing edible oral administration preparation of aggregated substancecontaining laminated film, and edible oral administration preparation of aggregated substance-containing laminated film, in, Google Patents, 2005.

[87] T. Awamura, 粟村努, H. Nishikawa, 西川久信, K. Kokaji, 小梶和彦, A. Ishise, 石瀬章浩, T. Arai, 荒井崇行, Film preparation containing medicament with unpleasant taste, in, Google Patents, 2011.

[88] T. Awamura, K. Furusawa, Y. Sawai, Rapidly soluble film preparation, in, Google Patents, 2005. 
[89] T. Awamura, Y. Sawai, Nicotine-containing film preparation, in, Google Patents, 2003.

[90] P. release, APR and Labtec's Ondansetron Rapidfilm ${ }^{\circledR}$ licensed to SciClone Pharmaceuticals for Chinese Market, in, Labtec GmbH, http://www.tesa-labtec.com/eng/company/press/apr-and-labtecs-ondansetronrapidfilm-licensed-to-sciclone-pharmaceuticals-for-chinese-market,3701696,1.html, 2009.

[91] P. Release, Launch of Setofilm ${ }^{\circledR}$ byNorgine in several European countries, in, http://www.tesalabtec.com/eng/company/press/tesa-labtec-tesa-labtec-and-apr-applied-pharma-research-apr-announce-thelaunch-of-setofilm-ondansetron-oral-dispersible-film-in-several-european-countries-by-norgine, 5163100,1.html, 2013.

[92] C. Leichs, A. Breitenbach, I. Lehrke, P. Galfetti, Non-mucoadhesive film dosage forms, in, Google Patents, 2008.

[93] G. Reiner, N. Giarratana, V. Reiner, U. Becker, A. Breitenbach, P. Klaffenbach, Ph regulating antibacterial films for the oral or vaginal cavity, in, Google Patents, 2010.

[94] P. Release, APR Applied Pharma Research and Labtec GmbH Announce Approval in Europe of Zolmitriptan Oral Dispersible Film (ODF), in, http://www.tesa-labtec.com/eng/company/press/apr-and-labtec-announceapproval-in-europe-of-zolmitriptan-rapidfilm,3701615,1.html, 2012.

[95] A. Breitenbach, N. Schwier, Oral film formulation, in, Google Patents, 2012.

[96] A. Breitenbach, N. Schwier, Rapidly disintegrating oral film formulation for olanzapin, in, Google Patents, 2012.

[97] A.D.K.P.S. Breitenbach A; Breitenbach, Nina Dr.; Klaffenbach, Peter Dr.; Schwier N Pharmaceutical preparation with improved stability of the active agent, in, Labtec GmbH 2010.

[98] PHARMAZIE, Orale Filme Auf die Zunge, fertig, los, PZ PHARMAZEUTISCHE ZEITUNG, 155 (2010).

[99] I. BioDelivery Sciences International, BEMA ${ }^{\circledast}$ Buprenorphine, in, http://www.bdsi.com/BEMA Technology.aspx, 2014.

[100] B.G. A, H. Richard, J. Scott, K. Kasey, O.D. W, P. Amy, Process for loading a drug delivery device, in, Google Patents, 2003.

[101] N. Vasisht, A. Finn, Transmucosal delivery devices with enhanced uptake, in, Google Patents, 2008.

[102] H. Richard, O.D. W, Adhesive bioerodible transmucosal drug delivery system, in, Google Patents, 2005.

[103] F. Zeng, L. Eleuterius, Hot melt extruded film containing silicon dioxide, in, Google Patents, 2009.

[104] H.Q. Mao, C.K. Yu, V.L. Truong, Y. Li, D. Rangaraj, X. Jiang, S.R. Shah, D.C. Sing, Quick-dissolving oral thin film for targeted delivery of therapeutic agents, in, Google Patents, 2010.

[105] C.K. Chu, J.P. Ryoo, Z. Wang, Dosage form for insertion into the mouth, in, Google Patents, 2012.

[106] W.-J.O.J.G.O. Choi, Joon-Gyo; Lee Yoon Jung; Lee, Yoon-Jung; Lee Bong Yong; Lee, Bong-Yong; Choi Na Young; Choi Won Jae; Choi, Na-Young, Film for oral administration containing mirodenafil or pharmaceutically acceptable salt thereof, in, SK Chemicals Co., Ltd. , 2013.

[107] Y.-J.Y.J.L.B.Y.L.J.G.O.C. Lee, Na-Young; Choi, Won-Jae; Na Yeong Choi; Oh, Joon-Gyo; Won Jae Choi; Lee, Bong-Yong Film for oral administration containing montelukast or pharmaceutically acceptable salt thereof, in, SK Chemicals Co., Ltd., 2013.

[108] AstraZeneca, Delivering value through innovation, in: A.A.R.a.F.-F.I. 2012 (Ed.), http://www.astrazenecaannualreports.com/2012/documents/eng download centre/annual report.pdf, 2012.

[109] AstraZeneca, Bioequivalence Study Comparing Arimidex Tablet and Anastrozole ODF in Japanese Healthy Male Subjects, in, Clinicaltrails.gov, http://clinicaltrials.gov/ct2/show/NCT01568281?term=Anastrozole+film\&rank=1, March 28, 2012.

[110] E. Ron, S.; Ron Eyal S; Farber, Neal M.; Farber, Neal, M.; Ron, Eyal S.; Merello Marcelo; Ron Eyal S.; Merello, Marcelo; Farber Neal M.; Farber Neal M Methods and compositions for decreasing saliva production, in, Neurohealing Pharmaceuticals, Inc., 2006.

[111] E.R. Ron, Yoram; Ron, Eyal S.; Cohen, Smadar New oral dissolving films for insulin administration, for treating diabetes, in, Pharmedica Ltd. , 2012. 
[112] A.J.B.N.J.G. Giovinazzo, Anthony, John; Bryson, Nathan, John; Barnhart Scott David; Koons, Michael, Clinton; Barnhart, Scott, David; Bryson, Nathan John; Koons, Michael Clinton; Koons Michael Clinton; Barnhart, Scott David; Giovinazzo Anthony John Sublingual films, in, Cynapsus Therapeutics, Inc.; Arx, Llc 2012.

[113] N.J.H.D.B.S.M.L.D.D.S.M.L.H.D.H. Bryson Nathan John; Bryson, David, Bruce; Hedden, David Bruce; De Somer, Marc L.; Giovinazzo Anthony John; Giovinazzo A; De Somer Marc L; Bryson N; De Somer, Marc, L.; Bryson N J; Hedden D B; Giovinazzo, Anthony, John; Bryson, Nathan, John; Giovinazzo, Anthony John; De Somer Marc L.; De Somer M; Giovinazzo A J Sublingual apomorphine, in, Adagio Pharmaceuticals Ltd.; Cynapsus Therpeutics 2010.

[114] M.A. Repka, Assessing THC Pro-drugs in Transmucosal Patch Delivery Formulations for the Treatment of Neuropathological Conditions, http://www.umc.edu/Administration/Centers and Institutes/Center for Psychiatric Neuroscience/repka rese arch.aspx.

[115] P. Newswire, Aoxing Pharmaceutical Company Announces Issuance of Chinese Patent for Midazolam Maleate Oral Film, in: Medical Pharmaceuticals, http://www.prnewswire.com/news-releases/aoxingpharmaceutical-company-announces-issuance-of-chinese-patent-for-midazolam-maleate-oral-film-

124082189.html, 2011.

[116] P. Newswire, Aoxing Pharmaceutical Company Announces Issuance of Chinese Patent for Naloxone Sublingual Film, in: Pharmaceuticals, PR Newswire, http://www.prnewswire.com/news-releases/aoxingpharmaceutical-company-announces-issuance-of-chinese-patent-for-naloxone-sublingual-film-121080844.html,

2011.

[117] J.K.P. Jung K T; Tae Jung Kyoung; Yang Won-Suk; Jung Kyoung-Tae; Yang W S; Tae Jeong G; Park, Jin-Kyu; Gyu Park J; Park J; Park J K; Park Jin-Kyu; Jung, Kyoung Tae; Park Jin Kyu; Jung Kyoung Tae; Yang, Won Suk; Yang Won Suk; Suk Yang Won; Jung K; Kyu Park Jin; Yang, Won-Suk; Seok Yang W; Yang W Fast-dissolving oral film for effectively concealing unpleasant tastes, in, Bexcore Inc. , 2010.

[118] C.W.J.C.-W.L.S.M.H.Y.M.J.C.W.J. Jun, Kyoung Tae; Jung Kyoung-Tae; Jung Kyoung Tae Stable fast-dissolving film formulation for oral dosage form, in, Chabio \& Diostech Co., Ltd. , 2012.

[119] Y.S.S. Kim, Jun Ho Fast-dissolving oral film preparation comprising aripiprazole, in, CHA Bio \& Diostech Co., Ltd. , 2014.

[120] E.K. Yoon, CTC Bio works for orally-disintegrating clomipramine in: English Dailypharm News korean pharmaceutical news, http://www.dailypharm.com/Users/News/EnglishNews.html?NewsID=3556\&nStart=33\&mode=\&searchValue=, 2013.

[121] J.-H.L. Yoo, Sun-Ahe; Lee, Bong-Sang; Kwak, Seong-Shin; Kwon, Do-Woo; Jeon, Hong-Ryeol; Park, HyunJung Pharmaceutical composition for treating premature ejaculation and method for treating premature ejaculation, in, CTC Bio, Inc. , 2013.

[122] H.-R.P. Jeon, Su-Jun; Kim Jun Ki; Cha, Bong-Geun; Park Su Jun; Kim, Jun-Ki; Lee, Bong-Sang; Jeon Hong Ryeol; Cha Bong Geun; Lee Bong Sang Sildenafil-free base-containing film preparation and method for producing same, in, Ctc Bio, Inc. , 2012.

[123] P. Newswire, CURE Pharmaceutical to Begin Clinical Trials with PediaSUNATE(TM), in, http://www.prnewswire.com/news-releases/163534016.html, 2012.

[124] K. Stenberg, F. Huebinette, Water-soluble films comprising low-viscosity alginates, in, Google Patents, 2007.

[125] F. Hübinette, Nicotine formulation, in, Google Patents, 2013.

[126] Aavishkar, Innovation for healthier life... in, http://www.aavishkar.org/r d.html, 2013.

[127] G.S. Rekhi, Novel technologies improve oral drug delivery performance, in: Formulation (Ed.) The Leader of the Pack, Pharmaceutical Formulation \& Quality, http://www.pharmaquality.com/me2/Audiences/dirmod.asp?nm=Browse+Articles\&type=Publishing\&mod=Publ 
ications\%3A\%3AArticle\&mid=D3E3C719D8D44216836DCA4F4144BEC4\&tier=4\&id=41C41043EA4C451DBC2874 3E31E04755\&AudID=5648A5C28C97462DBBDB309539B820EF, 2009.

[128] D.M. Bajaj A, Challenges and strategies in novel drug delivery technologies, Pharma Times, 38 12-16.

[129] Reportlinker, Drug Delivery Technology Industry Market Research \& Statistics, in: Global Drug Delivery Technology Industry, ReportLinker.com http://www.reportlinker.com/ci02258/Drug-Delivery-Technology.html, 2015.

[130] M.K. Banbury S, Fast-dispersing dosage forms for pedriatic market, Pedriatic delivery, 2 (2011) 32-35.

[131] C. WOODLAND HILLS, Zengen's new technology first to deliver drug active ingredients in an oral strip, in, Bio-Medicine, $\quad$ http://news.bio-medicine.org/biology-news-2/Zengens-new-technology-first-to-deliver-drugactive-ingredients-in-an-oral-strip-3916-1/, 2003.

[132] N.J. PARSIPPANY, Triaminic and Theraflu Thin Strips Receive 'Best Product of 2004 Award', in: Awards, PR Newswire, PRNewswire, 2004.

[133] I. Novartis Consumer Health, Triaminic Thin Strips ${ }^{\circledR}$ Day Time Cold \& Cough, in: Triaminic, Novartis, http://www.triaminic.com/products/thin-strips/day-time-cold-and-cough.shtml.

[134] M.R. Cohen, Orally dissolving film strips could pose hazard to kids, in: Check-up, Phylly.com, http://www.philly.com/philly/blogs/healthcare/Orally-dissolving-film-strips-could-pose-hazard-to-

kids.htm|\#IBrdBtip9FwbCtkX.99, 2013.

[135] M.L. Buck, Alternative Forms of Oral Drug Delivery for Pediatric Patients, PEDIATRIC PHARMACOTHERAPY, 19 (2013).

[136] M. Freedman, Unintentional ingestion of cough and cold medicines has declined, in: Contemporary Pediatrics, http://contemporarypediatrics.modernmedicine.com/print/378207, 2014.

[137] D.B.N. Lee M. Hampton, Jonathan R. Edwards and Daniel S. Budnitz, Cough and Cold Medication Adverse Events After Market Withdrawal and Labeling Revision, Pediatrics, 132 (2013) 8.

[138] IBISWorld, Thin Film Drug Manufacturing in the US: Market Research Report, in: Industry trends, IBISWorld, http://www.ibisworld.com/industry/thin-film-drug-manufacturing.html, 2012.

[139] N.J. WARREN, MonoSol Rx Announces Availability of Zuplenz ${ }^{\circledR}$ Oral Soluble Film for Licensing to New Commercialization Partners, in: Pharmaceuticals, PRNewswire, http://www.prnewswire.com/newsreleases/monosol-rx-announces-availability-of-zuplenz-oral-soluble-film-for-licensing-to-new-

commercialization-partners-125341993.html, 2011.

[140] V. Richmond, Reckitt Benckiser Pharmaceuticals Inc. Receives FDA Approval for Suboxone ${ }^{\circledR}$ (Buprenorphine and Naloxone) Sublingual Film C-III, in: RB Press Release, Reckitt Benckiser Pharmaceuticals Inc, http://www.rb.com/fda-approval-for-suboxone-sublingual-filmc-iii, 2010.

[141] Orally Disintegrating Tablets and Thin Films Poised to See Strong Growth, in, PRNewswire, April 18, 2013.

[142] I. BioDelivery Sciences International, BUNAVAIL ${ }^{\mathrm{TM}}$ (Buprenorphine/Naloxone buccal soluble film), in, http://www.bdsi.com/Other BEMA Products.aspx, 2014.

[143] R.B.P. Inc, Our Relationships and Principal Risks, in: RB Annual Report 2013, 2013, pp. 93-100.

[144] D. Sheon, New Drug Delivery Options that Help the Medicine Go Down, in: R.W.H. care (Ed.), http://www.realworldhealthcare.org/2013/05/new-drug-delivery-options-that-help-the-medicine-go-down/,

2013.

[145] CSNews, CSN - 2013 Industry Report - Reversal of Fortune, in, CSnews, http://www.csnews.com/articlecsn 2013 industry report reversal of fortune-5866.html, 2013.

[146] Pfizer, Pfizer Consumer Healthcare, in, http://archive.is/X0a2Y, 2005-2006.

[147] D.S. News, Pfizer.(OTC Editor's Picks)(Brief Article), in: $H$. Business (Ed.), http://business.highbeam.com/413375/article-1G1-134165166/pfizer.

[148] u.H. LLC, Sudafed PE Non-Drowsy Quick Dissolve Strips Cherry Menthol 10 / Box, in, http://ucanhealth.com/local/merchandise/?pid=181431\&ptt=Sudafed PE Non-

Drowsy Quick Dissolve Strips Cherry Menthol $10 /$ Box. 
[149] A.M. Traas, T. Fleck, A. Ellings, S. Mahabir, K. Stuebner, D.C. Brown, D. Durso, M. DiGregorio, L. Bode, K.I. Kievit, R. McCall, Ease of oral administration and owner-perceived acceptability of triglyceride oil, dissolving thin film strip, and gelatin capsule formulations to healthy cats, American journal of veterinary research, 71 (2010) 610-614.

[150] ScholarlyEditions, University of Pennsylvania, Philadelphia: Ease of oral administration and ownerperceived acceptability of triglyceride oil, dissolving thin film strip, and gelatin capsule formulations to healthy cats in: S.T. eBook (Ed.) Issues in Veterinary Research and Medicine: 2011 Edition, ScholarlyEditions, TM Atlanta, Georgia, http://books.google.pt/books?id=WriZdX0hYdkC\&pg=PT1051\&lpg=PT1051\&dq=veterinary+thin+films\&source= bl\&ots=Zmn9bS9Hf6\&sig=XFhf66Nd2HR5 Th3CnXAwP74leY\&hl=en\&sa=X\&ei=1SLyUoW OIWq0QX5wYDgCg\&ve $\mathrm{d}=0$ CEYQ6AEwBA\#v=onepage\& $\mathrm{q}=$ veterinary\%20thin\%20films\&f=false, 2012, pp. 1620 pages.

[151] V. Guru, Rapid Dissolve Pet Strips, in, Vet Guru, http://www.vetguru.com/product-category/rapid-dissolvepet-strips/, 2013.

[152] P. Wellness, Dogs Hate Pills, in, Pace Wellness, http://pacewellness.com/dogs-hate-pills.

[153] ReportLinker, Global Veterinary Industry, in: Veterinary healthcare Industry: Market Research Reports, Statistics and Analysis, http://www.reportlinker.com/ci02247/Veterinary-healthcare.html, 2014.

[154] M. Luke, 5 Reasons to Invest in Animal Health, in, http://www.fool.com/investing/general/2013/08/28/5reasons-to-invest-in-animal-health-care.aspx, 2013.

[155] G.I. Analysts, Animal health market to hit $\$ 43$ billion in five years, in: Management, Western Farm Press, http://westernfarmpress.com/management/animal-health-market-hit-43-billion-five-years, 2012.

[156] Freedonia, Animal Health Products to 2016 - Demand and Sales Forecasts, Market Share, Market Size, Market Leaders, in, http://www.freedoniagroup.com/Animal-Health-Products.html, 2013.

[157] L. Wang, R.L. Coppel, Oral vaccine delivery: can it protect against non-mucosal pathogens?, Expert review of vaccines, 7 (2008) 729-738.

[158] M.M. Levine, Immunogenicity and efficacy of oral vaccines in developing countries: lessons from a live cholera vaccine, BMC biology, 8 (2010) 129.

[159] L.i.t. matter, Global Human Vaccine Industry 2013-2018: Trend, Profit, and Forecast Analysis, April 2013, in, $\quad$ http://www.lucintel.com/reports/medical/global human vaccine industry 2013-

2018 trend profit and forecast analysis.aspx.

[160] B. Wire, Research and Markets: 2014 Report of the International Therapeutic Vaccines Market - Forecasts to 2018, in, CNBC, http://www.cnbc.com/id/101769995\#. 2014.

[161] E.I. AG, EUDRAGIT ${ }^{\circledR}$ Acrylic polymers for solid oral dosage forms, in: E.R. GmbH (Ed.), http://eudragit.evonik.com/product/eudragit/Documents/evonik-brochure-eudragit-product.pdf, 2014.

[162] P. Sneiderman, Students Devise Oral Quick-Dissolve Strips for Rotavirus Vaccine, in: Headlines@hopkins, Headlines@hopkins, http://www.jhu.edu/news/home07/may07/rotaviru.html, 2017.

[163] Clinicaltrials.gov, The Safety and Efficacy of a Peanut Immunotherapy Dissolving Film for Peanut Allergy, in: NCT01897077, July 8, 2013, http://clinicaltrials.gov/ct2/show/NCT01897077?term=peanut+film\&rank=1, 2013.

[164] R. Wood, H.Q. Mao, C. KEET, R. Martin, Orally dissolving thin films containing allergens and methods of making and use, in, Google Patents, 2013.

[165] E. Jones, E. Ojewole, R. Kalhapure, T. Govender, In vitro comparative evaluation of monolayered multipolymeric films embedded with didanosine-loaded solid lipid nanoparticles: a potential buccal drug delivery system for ARV therapy, Drug development and industrial pharmacy, 40 (2014) 669-679.

[166] C. Shen, B. Shen, H. Xu, J. Bai, L. Dai, Q. Lv, J. Han, H. Yuan, Formulation and optimization of a novel oral fast dissolving film containing drug nanoparticles by Box-Behnken design-response surface methodology, Drug development and industrial pharmacy, 40 (2014) 649-656.

[167] P. Rana, R.S. Murthy, Formulation and evaluation of mucoadhesive buccal films impregnated with carvedilol nanosuspension: a potential approach for delivery of drugs having high first-pass metabolism, Drug Deliv, 20 (2013) 224-235. 
[168] C. Dott, C. Tyagi, L.K. Tomar, Y.E. Choonara, P. Kumar, L.C. du Toit, V. Pillay, A Mucoadhesive Electrospun Nanofibrous Matrix for Rapid Oramucosal Drug Delivery, Journal of Nanomaterials, 2013 (2013) 19.

[169] C. Cavallari, A. Fini, F. Ospitali, Mucoadhesive multiparticulate patch for the intrabuccal controlled delivery of lidocaine, European journal of pharmaceutics and biopharmaceutics : official journal of Arbeitsgemeinschaft fur Pharmazeutische Verfahrenstechnik e.V, 83 (2013) 405-414.

[170] J.O. Morales, A.C. Ross, J.T. McConville, Protein-coated nanoparticles embedded in films as delivery platforms, Journal of Pharmacy and Pharmacology, 65 (2013) 827-838.

[171] C. Giovino, I. Ayensu, J. Tetteh, J.S. Boateng, Development and characterisation of chitosan films impregnated with insulin loaded PEG-b-PLA nanoparticles (NPs): A potential approach for buccal delivery of macromolecules, International Journal of Pharmaceutics, 428 (2012) 143-151. 